\title{
Influence of Money Distribution on Civil Violence Model
}

\author{
Ignacio Ormazábal, ${ }^{1,2}$ F. A. Borotto, ${ }^{1,2}$ and H. F. Astudillo ${ }^{1,2}$ \\ ${ }^{1}$ Departamento de Física, Universidad de Concepción, Concepción, Chile \\ ${ }^{2}$ Grupo de Sistemas Complejos, Universidad de Concepción, Concepción, Chile \\ Correspondence should be addressed to H. F. Astudillo; hastudil@udec.cl
}

Received 7 July 2017; Accepted 9 October 2017; Published 5 November 2017

Academic Editor: Dimitri Volchenkov

\begin{abstract}
Copyright (C) 2017 Ignacio Ormazábal et al. This is an open access article distributed under the Creative Commons Attribution License, which permits unrestricted use, distribution, and reproduction in any medium, provided the original work is properly cited.
\end{abstract}

\begin{abstract}
We study the influence of money distribution on the dynamics of Epstein's model of civil violence. For this, we condition the hardship parameter distributed according to the distribution of money, which is a local parameter that determines the dynamics of the model of civil violence. Our experiments show that the number of outbursts of protest and the number of agents participating in them decrease when the distribution of money guarantees that there are no agents without money in the system as a consequence of saving. This reduces social protests and the system shows a phase transition of the second order for a critical saving parameter. These results also show three characteristic regimes that depend on the savings in the system, which account for emerging phenomena associated with the saving levels of the system and define scales of development characteristic of social conflicts understood as a complex system. The importance of this model is to provide a tool to understand one of the edges that characterize social protest, which describes this phenomenon from the sociophysics and complex systems.
\end{abstract}

\section{Introduction}

In the last decades, different studies have been carried out that seek to describe the society in the framework of complex systems [1]. One of the most used modeling approaches is the so-called bottom-up [2]. This approach is characterized by the use of agent-based models (MBAs) to reproduce artificial worlds or virtual societies and has been widely used to describe sociological phenomena such as the dynamics of segregation [3] and cultural diffusion [4]. A further review of these topics is found in the books of Epstein $[5,6]$.

Social conflicts have been characterized as emergent properties of a complex system that depends on the scale and levels at which they occur [7], resulting in protests, civil violence, wars, or revolutions. The use of variations of the model proposed by Epstein [8] has allowed describing different scenarios of social unrest, such as workers' protests due to wage inequality [9], propagation and persistence of criminal activity in the population [10], or cases of civil war between ethnic groups due to their geographical distribution [11]. Moreover, authors have worked on variants of the Epstein model [8] that have led to increasing the complexity of the dynamics. A class of models with this characteristic is achieved by adding more variables to the dynamics. Other models incorporate strategies into agents' decisions, such as allowing agents to gain learning using game theory or collective coevolution given by the interaction between agents [12]. There are also models where one of the parameters that define dynamics, such as legitimacy, has endogenous feedback [13].

To interpret the results obtained in the simulations, it is appropriate to use the concepts and tools of statistical physics. This fact has given rise to branches or new fields of interdisciplinary research [14-16] such as the econophysics [17-20] and the sociophysics [21-23]. Econophysics studies and describes statistical properties of economic systems with large amounts of economic and financial data through the use of techniques and tools originally developed in statistical physics [18]. In sociophysics the social behavior is described making use of the physics of the critical phenomena, emphasizing, among these, the use of the theory of phase transitions to describe social, psychological, political, and economic phenomena [24-27].

The goal in this paper is to study the dynamics of civil violence when the money distribution is taken into consideration. For this we condition the hardship parameter distributed according to the money distribution, unlike the 
Epstein model [8] where it is uniformly distributed. The distribution of money is a result of the dynamics of money exchange between agents with a propensity to save money, as obtained in $[28,29]$. In this way, we can study the influence of inequality in the money distribution of the society in the emergence of social mobilizations.

The order in which the contents are presented will be as follows. In Section 2, we will briefly describe the models used for the simulations and the elements that compose the comparative study. Then, in Section 3, we present the results obtained; then, in Section 4, we conduct the discussion and establish the conclusions.

\section{Civil Violence Model}

This agent-based model [8] simulates a process of social protest to which authority responds with the use of force to restore public order. The dynamic is established by relating the legitimacy of authority and the hardship of agents to the discontent of the population. The general state of the system is determined by global variables such as the legitimacy $L$, the vision of the agents $v$, the maximum sentence $J_{\max }$, and a state evaluation threshold $T$.

The agents, citizens, and police are placed at random on a rectangular grid with periodic edge conditions. The dynamics of the agents is achieved with decisions made taking into account the information obtained within the neighborhood of each agent selected. The neighborhood extension is defined by the range of vision of each agent. Citizens are agents that are characterized by two parameters: hardship $h$ and risk aversion $R$. These quantities are assigned to the agents as uniformly distributed random values. With these parameters and according to the evaluation of local conditions, each agent will decide whether or not to join the protest. To do this, each agent evaluates its state by means of its grievance $G=$ $h(1-L)$ and its net risk $N=R P$, where $P=1-\exp \left[-k(C / A)_{v}\right]$ is the probability of arrest that is obtained based on a number of active agents $A$ and police $C$ in your neighborhood.

Thus, the active state indicates that the agent joins the protest and the passive state is when the agent stays on the sidelines. On the other hand, the police are agents that do not have parameters assigned and are responsible for restoring order by capturing the active agents who are in their neighborhood defined by their vision.

The rules that determine the dynamics of the model are the following:

(1) Rule of motion: it is valid for all agents and allows them to move to an empty space at random within their neighborhood.

(2) The rule of state evaluation: it is given by $G-N>$ $T$. Citizens who comply with this rule transform their status from a passive agent to an active agent; otherwise, the agent will remain a passive agent.

(3) Capture rule: cops randomly capture an active agent from among the active agents in their neighborhood and move to that location on the grid. In case there are no active agents, they do nothing.
Epstein, to determine the existence of emerging phenomena, which he defines as "macroscopic regularities arising from the pure local interaction of agents" [2], proposes to study the waiting times distribution between each outburst and distribution of their sizes.

For the study of waiting times, Epstein defines a threshold of 50 active agents to consider the event as an outburst, where the outburst size is defined as the maximum amount of active agents during the event. Based on simulations carried out with $10^{5}$ iterations, Epstein finds that the waiting times distribution corresponds to a log-normal distribution, while the outburst size distribution corresponds to a Weibull distribution [8].

Table 1 lists the global and local parameters used to reproduce the results reported by Epstein [8]. The size of the grid is $(40 \times 40)$ and has toroidal topology, agent density is 0.7 , police density is 0.04 , and the probability of arrest is $k=2.3$.

Figure 1 shows the results obtained when reproducing the Epstein [8] model. Panel (a) shows the temporal evolution of the number of agents participating in a protest. The red line represents the number of active agents in the system. The observed events correspond to the phenomenon of punctuated equilibrium [30]. In panel (b), the histogram of the waiting times is displayed, while in panel (c), the histogram of the outburst sizes is displayed. For these results, we considered 50 experiments of $10^{5}$ iterations and the parameters shown in Table 1.

\section{Money Distribution Models}

At the end of the 90s, studies were conducted on the distribution of money, income, and wealth to describe the dynamics of economic systems by modeling commercial transactions such as the interactions between the molecules of a gas where they exchange momentum and energy. Therefore, they are appropriately described by the use of tools of statistical physics [18-20].

In particular, the results obtained with gas-like models $[28,29,31,32]$ have allowed the researchers to establish a parallel with a thermodynamic system closed in thermal equilibrium, where the agents exchange an amount of money as the particles of a gas in a closed system exchange momentum and energy. This analogy is possible since ordinary economic agents can only exchange money with other agents. They are not allowed to "make" money, for example, printing dollar bills [32]. In a system of agents that perform exchanges that fulfill the condition of time reversal, the distribution of money corresponds to the distribution of Boltzmann-Gibbs [32]. With these considerations, we can use the canonical ensemble to perform the physical description and obtain the probability function associated with an amount of money and with this know the distribution of this in the system.

In this paper, we use the results obtained from the dynamics of money exchange in the case that all agents save a portion of their money at the moment of making a money transaction and of the remaining amount the agents exchange a random fraction of it [28].

Thus, we have a system with total money $M$ and $N$ agents, which initially have an equal amount of money. The saving 


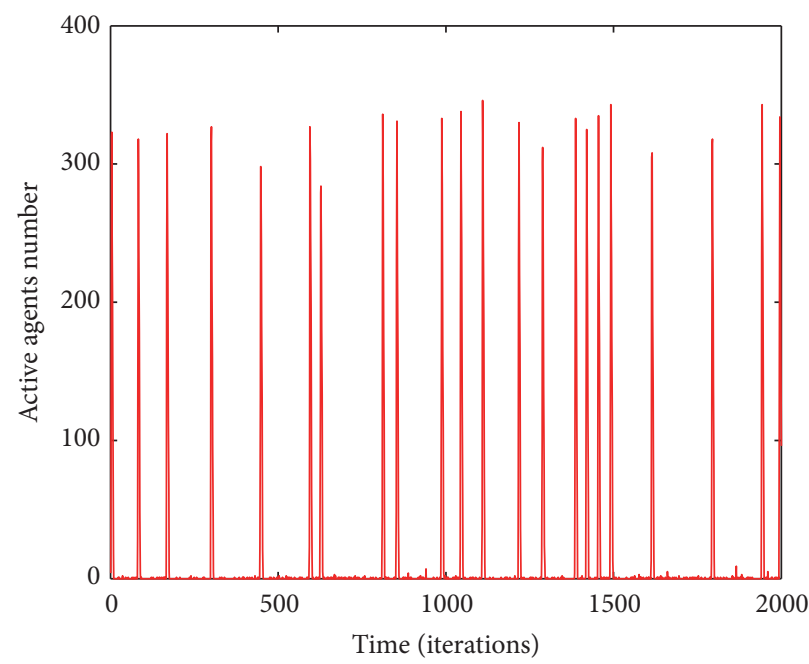

(a)

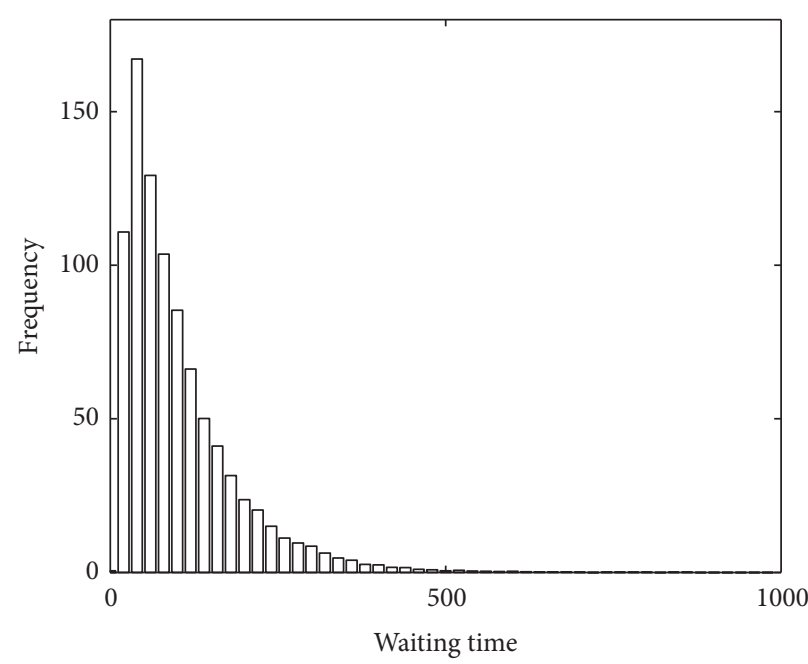

(b)

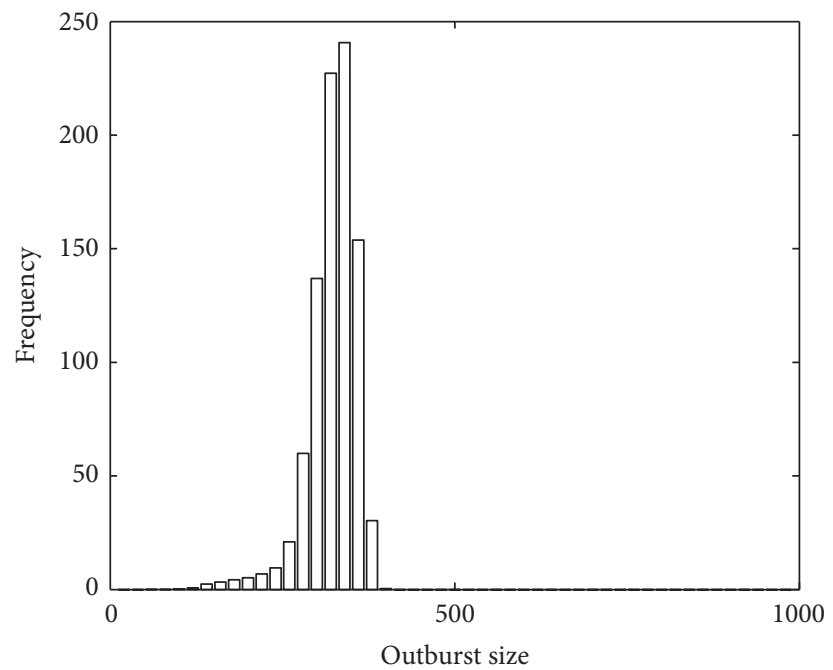

(c)

FIGURE 1: Results obtained when reproducing the model of Epstein [8]. Panel (a) shows the temporal evolution of the number of agents participating in a protest. The red line represents the number of active agents in the system. The observed events correspond to the phenomenon of punctuated equilibrium [30]. In panel (b), the histogram of the waiting times is displayed, while in panel (c), the histogram of the outburst sizes is displayed. For these results, we considered 50 experiments of $10^{5}$ iterations and the parameters shown in Table 1.

TABLE 1: Global and local parameters used to reproduce the results reported by Epstein [8]. The size of the grid is (40 $\times 40)$ and has toroidal topology, agent density is 0.7 , police density is 0.04 , and the probability of arrest is $k=2.3$.

\begin{tabular}{lcclcr}
\hline & Global parameters & Value & Local parameters & Value \\
\hline$v$ & Vision & 7 & $h$ & Hardship & $h \sim U[0,1]$ \\
$L$ & Legitimacy & 0.82 & $R$ & Risk aversion & $R \sim U[0,1]$ \\
$T$ & Threshold & 0.10 & & & \\
$J_{\max }$ & Maximum sentence & 30 & & & \\
\hline
\end{tabular}

factor is homogeneous in the system. The exchange of money between agents obeys the equation $\left[m_{i}, m_{j}\right] \rightarrow\left[m_{i}^{\prime}, m_{j}^{\prime}\right]=$ $\left[m_{i}-\Delta m, m_{j}+\Delta m\right]$, where the fraction $\Delta m$ being exchanged is given by

$$
\Delta m=(1-\lambda)\left[m_{i}-\epsilon\left(m_{i}+m_{j}\right)\right],
$$

where $\epsilon$ is a random number with a uniform distribution between zero and unity. Note that when the agents do not save $(\lambda=0)$, we retrieve the Boltzmann-Gibbs distribution obtained in the work of Dragulescu and Yakovenko [32]. When the saving is different from zero $(\lambda \neq 0)$, a money distribution is similar to the gamma distribution [31]. 
Second, we will use the description of Chatterjee et al. [29], in which the saving factor $\lambda$ is different for all agents and evenly distributed, that is, a heterogeneous factor in the system. In this case, the fraction $\Delta m$ being exchanged is given by

$$
\Delta m=\epsilon\left(1-\lambda_{j}\right) m_{j}-\left(1-\lambda_{i}\right)(1-\epsilon) m_{i},
$$

where the saving factors for each agent $\lambda_{i}, \lambda_{j}$, and $\epsilon$ are uniformly distributed random values between 0 and 1 . The result of this configuration gives a money distribution given by a mixture between a Boltzmann-Gibbs distribution and a Pareto, which is very close to the results reported in the analysis made to real wealth distribution and income data [29, 33].

In Figure 2, the results obtained by reproducing the distributions of money obtained in $[29,31]$ are shown. In panel (a), the money distributions are shown for cases where saving is a homogeneous feature in the system. The blue circles show the boundary case with $\lambda=0.00$, which corresponds to an exponential distribution as reported in [32]. The cases with $\lambda=[0.10,0.40,0.70,0.90]$ are shown with circles of red, green, yellow, and magenta, respectively. These results correspond to a gamma distribution as reported in [31]. In panel (b), the money distribution for $\lambda$ distributed heterogeneously in the system is shown, which corresponds to a Pareto distribution as reported in [29]. To obtain these results, 1120 agents with initial money $m_{0}=1000$ and 30000 were considered.

\section{Epstein Model Conditioned by the Money Distribution of Agents with Propensity to Save}

In order to study the influence of the money distribution on the emergence of social mobilizations we modified the hardship parameter that in the model civil violence is evenly distributed through the following expression:

$$
h_{i}\left(m_{i}\right)=1-\frac{m_{i}}{m_{\max }},
$$

where $m_{i}$ is the amount of money of the agent $i$ and $m_{\max }$ is the amount of money of the richest agent in the system. Thus, the preparation of the numerical experiments with this hybrid model is carried out following the steps indicated below:

(1) It is assigned initial values for each parameter, both local and global.

(2) At the beginning of the procedure, 30000 economic exchanges are carried out between agents to obtain the money distribution. This number of iterations is above the number of iterations necessary to reach the constant entropy regime in the system. In this process of exchange of money, only the agents of citizen type participate. Agents with the role of police do not participate in the exchange of money nor do they evaluate the state nor participate in the protests.

(3) The distribution of hardship is obtained from the distribution of money by means of (3).

(4) The simulation of civil violence begins.
In this study to obtain the average results of each case shown, 50 experiments of 100000 iterations each were carried out. In our study, we consider two cases in relation to the legitimacy parameter, $L$ : the case with $L=0.82$ as in Epstein and the case with $L=0.89$. The latter corresponds to the value obtained after rescaling the legitimacy by taking the mean value of the state evaluation rule obtained with both the hardship uniform distribution parameter and the hardship parameter obtained with the money distribution. In Table 2, the parameters used in both cases are shown.

Following the analysis made for the model of civil violence, for each case, we will present the distributions of waiting times and sizes of the outbursts. To compare the results of the Epstein model with the present hybrid model, we used outburst quantity, waiting times, and outburst sizes.

\section{Results of the Simulations}

First, we perform simulations of the model conditioned by the distribution of money with the parameters of the original Epstein model (see Table 2).

In Figure 3(a), the change of the distribution of the waiting times according to the saving parameters $\lambda=[0.00$, $0.20,0.55,0.65,0.80,0.83]$ is shown, which are drawn with lines and circles of red, green, yellow, black, magenta, and cyan, respectively. The line with squares in blue corresponds to the case with heterogeneous saving in the system, while the line with blue circles corresponds to the distribution obtained with the original Epstein model. In Figure 3(b), the changes in the size distribution of the burst are shown for the saving parameters $\lambda=[0.00,0.20,0.55,0.65,0.80,0.83]$, which are drawn with lines and circles of red, green, yellow, black, magenta, and cyan, while the blue line with squares corresponds to the case with heterogeneous saving in the system. The blue line with circles corresponds to the Epstein model.

In Figure 3(a) it can be observed that, for the case of heterogeneous distribution of savings, the short waiting times are more frequent, the reason why the system is at its maximum activity. The same characteristic is observed for the case of zero saving, where the highest frequency is obtained for short times. As the saving parameter increases, the maximum frequency of the waiting times decreases progressively and occurs at similar waiting times greater than for cases of heterogeneous saving and zero saving. The dynamics obtained in the Epstein model is closer to cases of high savings factor. In Figure 3(b), as in the case of waiting times, cases of heterogeneous saving and zero saving have the maximum frequencies for large outburst sizes. As the saving parameter increases, the frequency maxima decrease and take place for smaller outburst sizes. For the case of Epstein, it is observed that the maximum of the frequencies is in outburst sizes similar to the case with saving $\lambda=0.80$.

In Figure 4(b), we observe how the waiting time between each burst increases as the saving increases. As in Figure 4(a), there is a strong increase in waiting times from $80 \%$ savings. For $\lambda=[0.85,0.87,0.90]$, no bursts are observed during the simulation time of $3 \times 10^{5}$ iterations.

In Figure 4(a), the outburst quantity is plotted according to the saving parameter. With the line with red squares, 


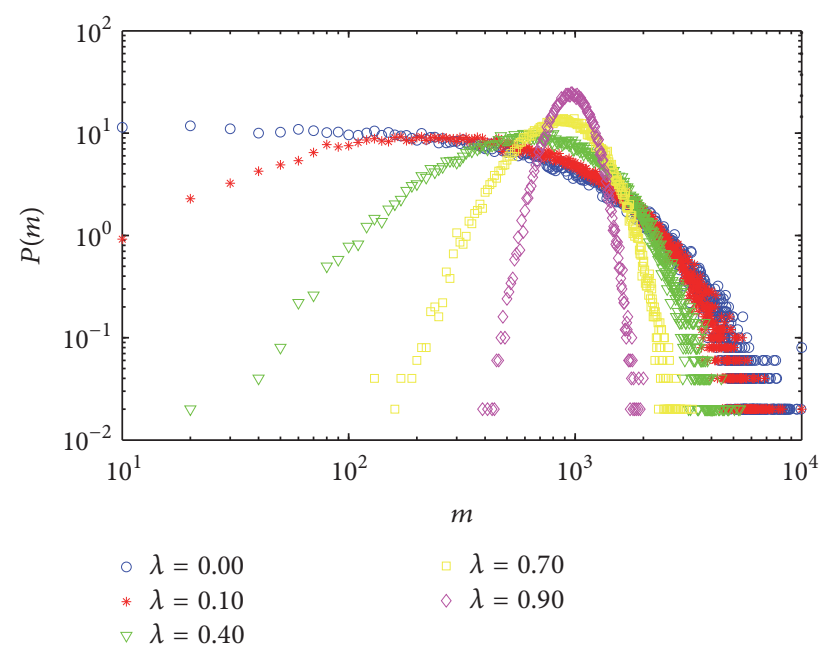

(a)

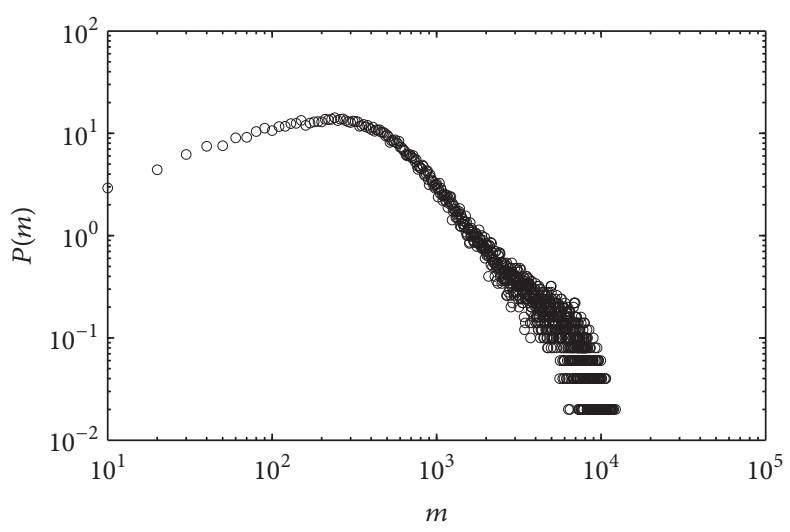

(b)

FIGURE 2: Results obtained by reproducing the distributions of money obtained in $[29,31]$. In panel (a), the money distributions are shown for cases where saving is a homogeneous feature in the system. The blue circles show the boundary case with $\lambda=0.00$, which corresponds to an exponential distribution as reported in [32]. The cases with $\lambda=[0.10,0.40,0.70,0.90]$ are shown with circles of red, green, yellow, and magenta, respectively. These results correspond to a gamma distribution as reported in [31]. In panel (b), the money distribution for $\lambda$ distributed heterogeneously in the system is shown, which corresponds to a Pareto distribution as reported in [29].

TABLE 2: Global and local parameters used in the simulations for Case I (a) and Case II (b). The grid dimensions are $40 \times 40$, its topology is toroidal, the agent density is 0.7 , the police density is 0.04 , and the arrest probability constant is $k=2.3$.

(a) Parameters for Case I

\begin{tabular}{lccccc}
\hline & Global parameters & Value & & Local parameters & Value \\
\hline$v$ & Vision & 7 & $h$ & Hardship & Given by Ec. (3) \\
$L$ & Legitimacy & 0.82 & $R$ & Risk aversion & $R \sim U[0,1]$ \\
$T$ & Threshold & 0.10 & $m_{o}$ & Initial money & 1000 \\
$J_{\max }$ & Maximum sentence & 30 & $\lambda_{\mathrm{I}}$ & Saving homogeneous & $\lambda_{\mathrm{I}} \in[0,0.90]$ \\
$t_{m}$ & Number of exchanges & 30000 & $\lambda_{\text {II }}$ & Saving heterogeneous & $\lambda_{\text {II }} \sim U[0,1]$ \\
\hline
\end{tabular}

(b) Parameters for Case II

\begin{tabular}{lcclcc}
\hline & Global parameters & Value & & Local parameters & Value \\
\hline$v$ & Vision & 7 & $h$ & Hardship & Given by Ec. (3) \\
$L$ & Legitimacy & 0.89 & $R$ & Risk aversion & $R \sim U[0,1]$ \\
$T$ & Threshold & 0.10 & $m_{0}$ & Initial money & 1000 \\
$J_{\max }$ & Maximum sentence & 30 & $\lambda_{\text {I }}$ & Saving homogeneous & $\lambda_{\text {I }} \in[0,0.35]$ \\
$t_{m}$ & Number of exchanges & 30000 & $\lambda_{\text {II }}$ & Saving heterogeneous & $\lambda_{\text {II }} \sim U[0,1]$ \\
\hline
\end{tabular}

the outburst quantity is shown. The blue circle corresponds to the value obtained with the Epstein model, while the green diamond corresponds to the value obtained for the case with heterogeneous savings. In Figure 4(b), the waiting times are plotted according to the saving parameter. With the line with red squares, the time between each outburst is plotted. The blue circle corresponds to the value obtained with the Epstein model and the green diamond corresponds to the value obtained for the case with heterogeneous savings. It is observed from Figure 4(a) that the outburst quantity decreases continuously as savings increase, approaching zero when $\lambda=\lambda_{c} \approx 0,87$, resulting in a phase transition. For cases of $\lambda=[0,87,0,90]$, the absence of outbursts is observed; that is, the punctuated equilibrium disappears giving rise to a regime that can be characterized as noise (as we shall see later), being the activity of active agents under the threshold for a burst. In Figure 4(b), we observe how the waiting time between each burst increases as the saving increases. As in Figure 4(a), there is a strong increase in waiting times from $80 \%$ savings. For $\lambda=[0.87,0.90]$, no bursts are observed during the simulation time of $3 \times 10^{5}$ iterations.

In Figure 5(a), the outburst sizes are plotted against the savings. The red lines show the average value of the size of the outburst in the range $\left[-2 \sigma_{\lambda}, 2 \sigma_{\lambda}\right]$, where $\sigma_{\lambda}$ is the standard deviation of the maximum values of the outburst sizes for each lambda value. The magenta and cyan 


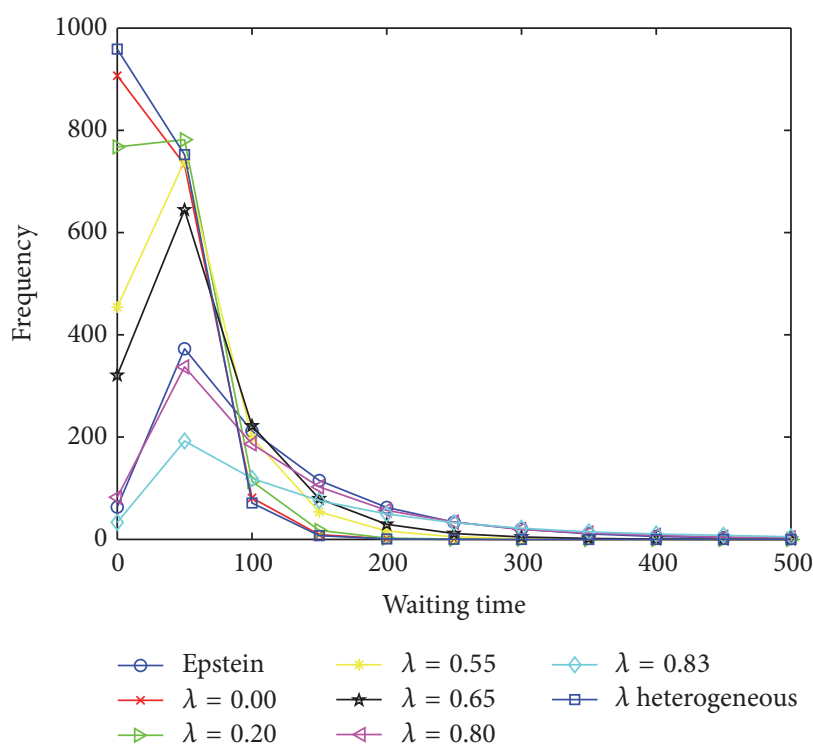

(a)

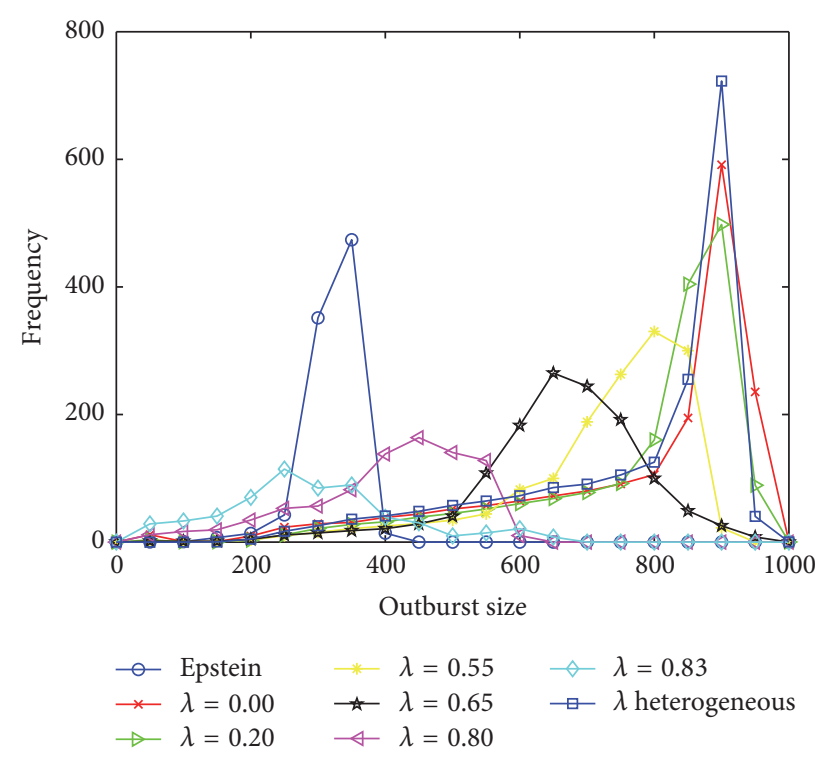

(b)

FIgURE 3: (a) Distribution of waiting times. The change of the distribution of the waiting times according to the saving parameters $\lambda=$ $[0.00,0.20,0.55,0.65,0.80,0.83]$ is shown, which are drawn with lines and circles of red, green, yellow, black, magenta, and cyan, respectively. The line with squares in blue corresponds to the case with heterogeneous saving in the system, while the line with blue circles corresponds to the distribution obtained with the original Epstein model. (b) Distribution of burst sizes. The changes in the size distribution of the burst are shown for the saving parameters $\lambda=[0.00,0.20,0.55,0.65,0.80,0.83]$, which is drawn with lines and circles of red, green, yellow, black, magenta, and cyan, while the blue line with squares corresponds to the case with heterogeneous saving in the system. The blue line with circles corresponds to the Epstein model.

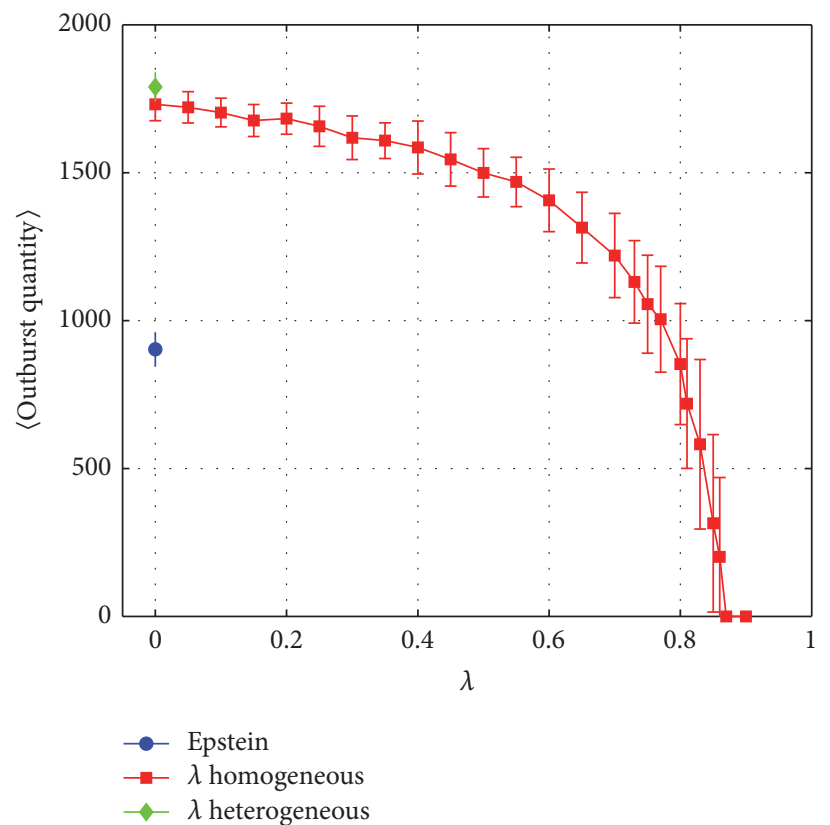

(a)

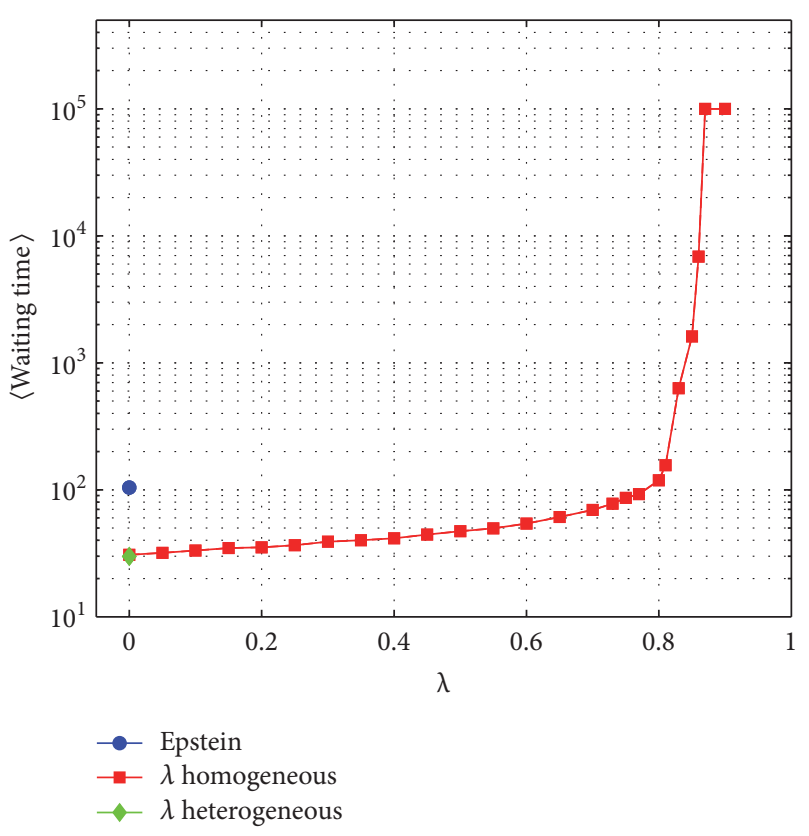

(b)

FIGURE 4: (a) The outburst quantity is plotted according to the saving parameter. With the line with red squares, the outburst quantity is graphed. The blue circle corresponds to the value obtained with the Epstein model, while the green diamond corresponds to the value obtained for the case with heterogeneous savings. (b) The waiting times are plotted according to the saving parameter. The line with red squares shows the time between each outburst for homogeneous $\lambda$. The blue circle corresponds to the value obtained with the Epstein model and the green diamond corresponds to the value obtained for the case with heterogeneous savings. 


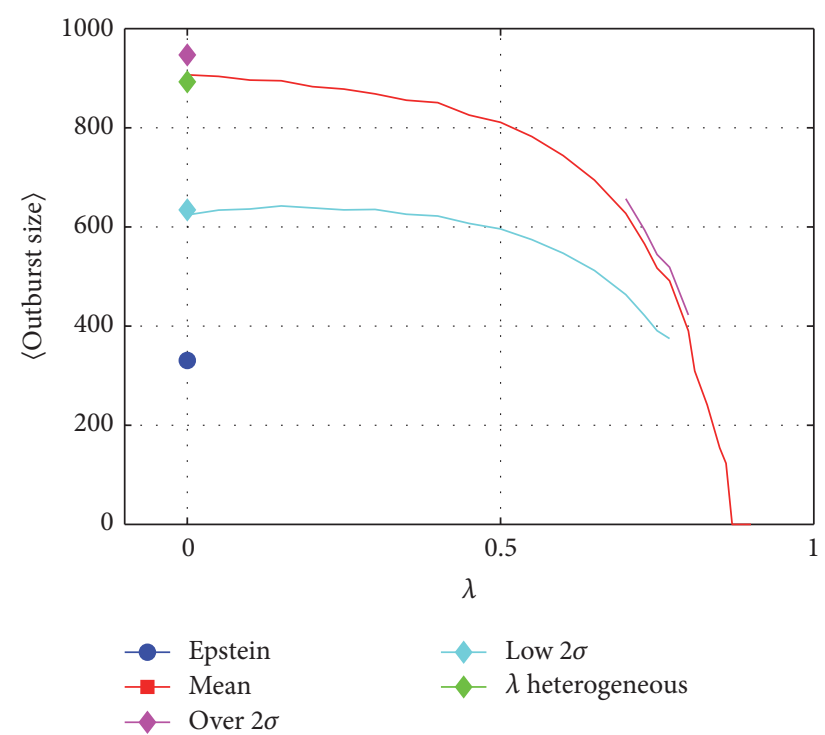

(a)

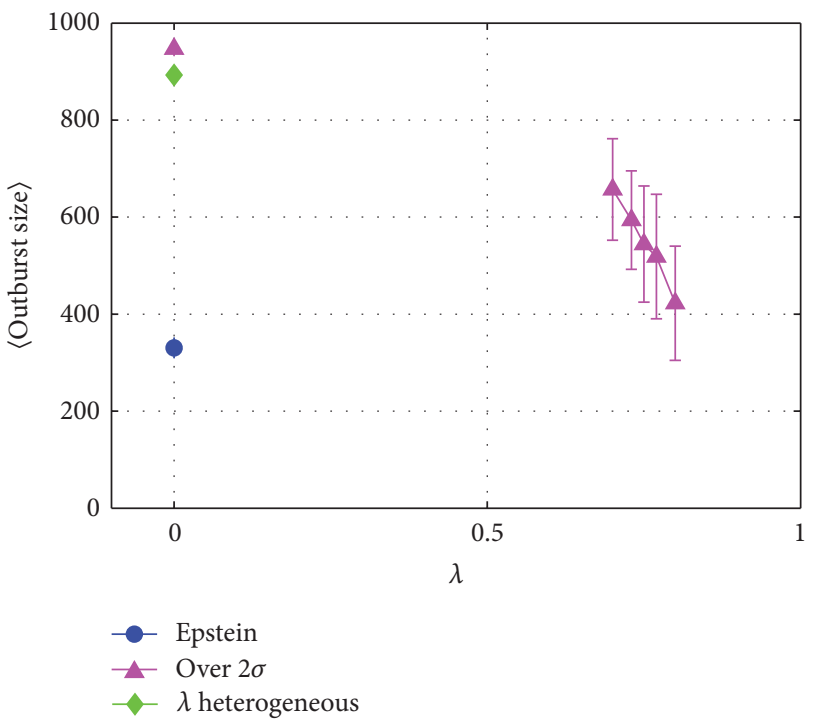

(c)

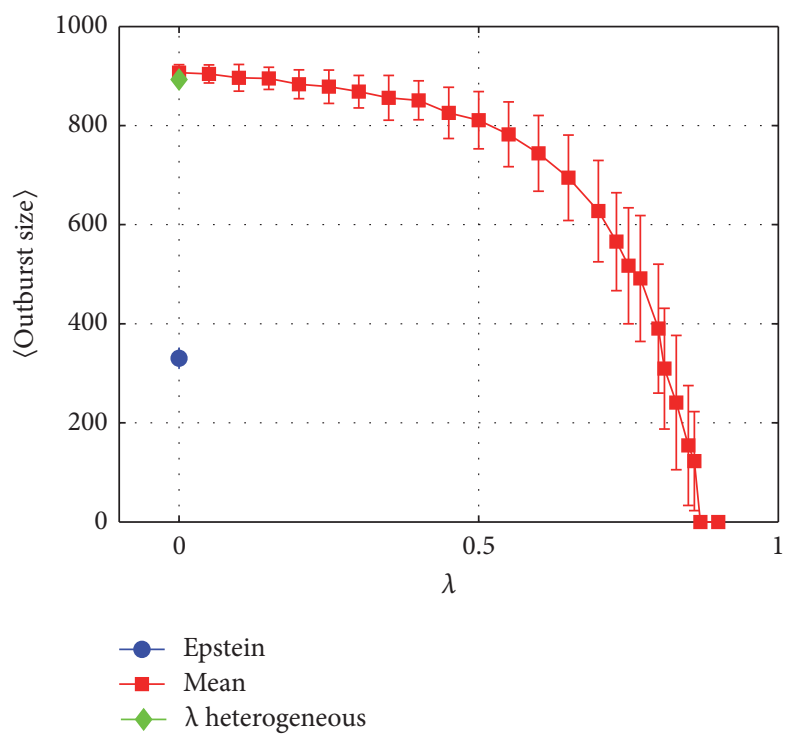

(b)

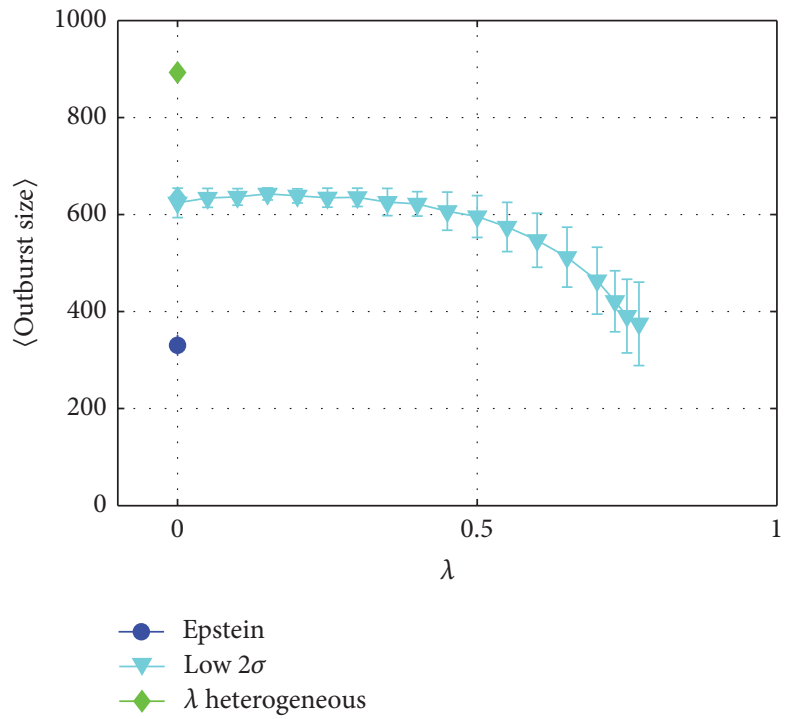

(d)

FIGURE 5: (a) The outburst size is plotted against the savings. The lines with red squares show the average value of the size of the outburst in the range $\left[-2 \sigma_{\lambda}, 2 \sigma_{\lambda}\right]$, where $\sigma_{\lambda}$ is the standard deviation of the maximum values of the outburst sizes for each lambda value. The magenta and cyan lines show the average value of the size of the outbursts above and below the range $\left[-2 \sigma_{\lambda}, 2 \sigma_{\lambda}\right]$, respectively. The blue circle corresponds to the average size of the outbursts obtained for the Epstein model and the diamond the average size of the outbursts obtained with heterogeneous lambda. In panels (b), (c), and (d), the average value of the size of the outbursts with their corresponding error bars, obtained both in the range $\left[-2 \sigma_{\lambda}, 2 \sigma_{\lambda}\right]$ and over and under the range, respectively.

lines show the average value of the size of the outbursts above and below the range $\left[-2 \sigma_{\lambda}, 2 \sigma_{\lambda}\right]$, respectively. The blue circle corresponds to the average size of the outbursts obtained for the Epstein model and the diamond the average size of the outbursts obtained with heterogeneous lambda. Figures 5(b), 5(c), and 5(d) show the average value of the size of the outbursts with their corresponding error bars, obtained both in the range $\left[-2 \sigma_{\lambda}, 2 \sigma_{\lambda}\right]$ and over and under the range, respectively.
As can be seen in Figure 5, the outburst size decreases similar to outburst quantity (see Figure 4) with the occurrence of three regimes for the maxima at each outburst. The lower intensity maxima (see Figure 5) correspond to outbursts that are not completely quenched, leaving a remnant of active agents large enough to generate a resurgence of the outburst, disappearing when $\lambda=0.77$. Outbursts with maximum values above range $\left[-2 \sigma_{\lambda}, 2 \sigma_{\lambda}\right]$ (see Figure 5(c)) appear as $\lambda$ approaches 0.7 and vanish as $\lambda$ approaches 0.80 . 


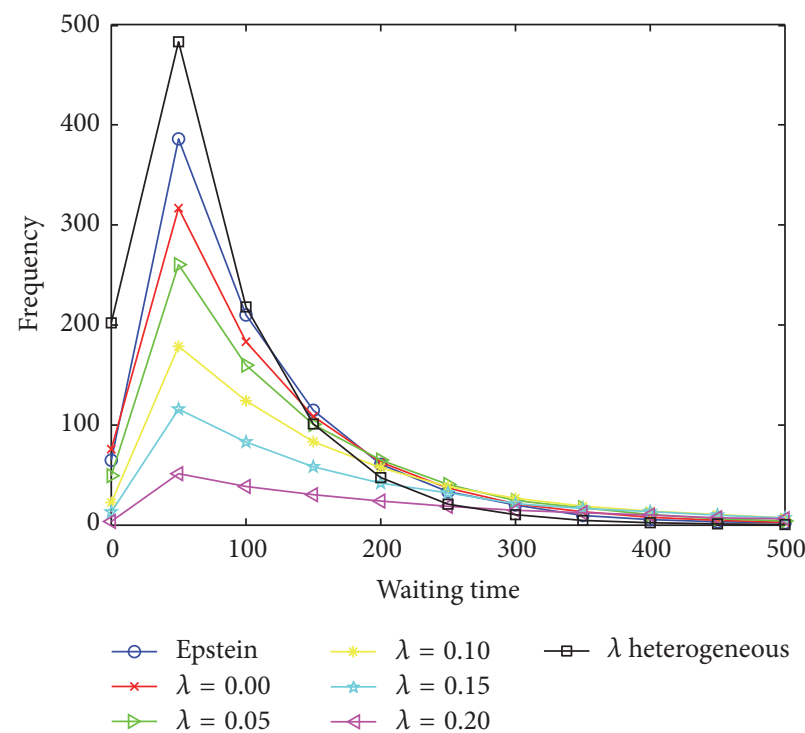

(a)

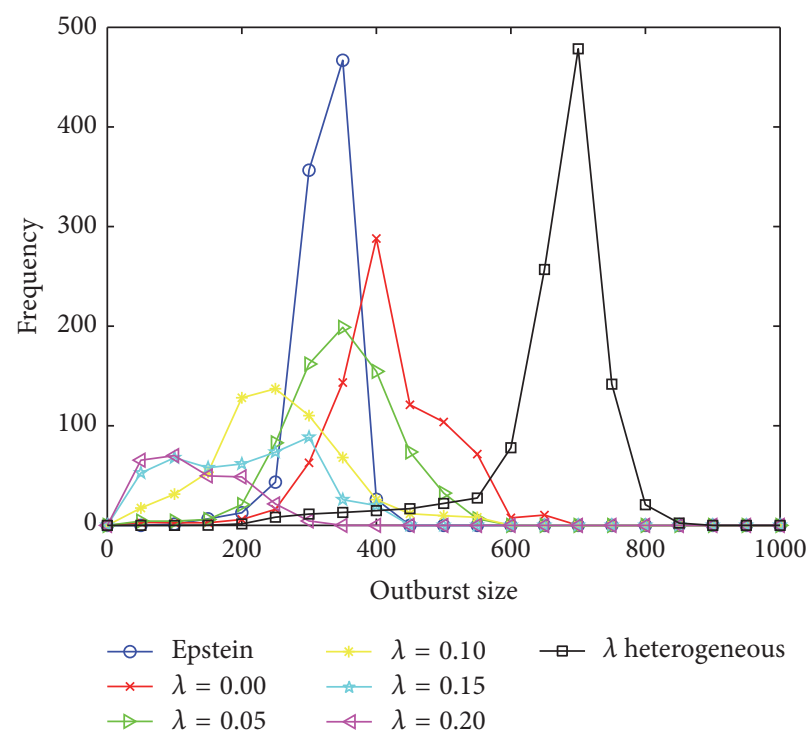

(b)

FIgURE 6: (a) The waiting time distribution with rescaled legitimacy. (b) The outburst sizes distribution with rescaled legitimacy for saving parameters is plotted. The cases with homogeneous saving are drawn with red, green, yellow, magenta, and cyan lines for the values, respectively. The line with squares in black corresponds to the case with heterogeneous saving in the system, while the line with blue circles corresponds to the distribution obtained with the original Epstein model.

The existence of these three regimens for the maxims of each outburst implies that the dynamics obtained with the model dependent on the distribution of money does not correspond rigorously to the punctuated equilibrium, as described by Bak and Sneppen [30] and Epstein [8].

The simulations performed so far use the legitimacy value of the original Epstein model. From Figures 3, 4, and 5, we can see that the sizes, the quantity of the outburst, and the waiting times for the case $\lambda=0.00$, that is, when the distribution of hardship is exponential, are notoriously greater than for the case of Epstein. The uniform hardship distribution has a smaller number of active agents than the exponential case. Clearly, the legitimacy of society in both cases cannot be the same.

In order to obtain a system with comparable dynamics to the Epstein model, in terms of outburst sizes, outburst quantity, and waiting times, we take the mean value of the equation that establishes the rule of state evaluation, to give

$$
\left\langle G_{E}\right\rangle=\left\langle G_{\lambda}\right\rangle \text {, }
$$

where $\left\langle G_{E}\right\rangle$ is the average grievance of the system for the model under the parameters of Epstein and $\left\langle G_{\lambda}\right\rangle$ is the average grievance for the savings-dependent system $\lambda$. Then,

$$
L_{\lambda}=1-\left[\frac{\left\langle h_{E}\right\rangle}{\left\langle h_{\lambda}\right\rangle}(1-L)\right] \text {. }
$$

Equation (5) allows us to obtain a value of the rescaled legitimacy that gives us the possibility of making the dynamics of the systems under discussion comparable. In Table 3, the average value of the hardship, $\left\langle h_{\lambda}\right\rangle$, and the rescaled legitimacy, $L_{\lambda}$, are shown for each saving factor value, $\lambda$. The value used in Epstein for legitimacy is $L=0.82$ and the mean value of the hardship is $\langle h\rangle=0.5$ ( $h$ is a random variable evenly distributed between 0 and 1 ). From Table 3 , it is read that the rescaling value of the appropriate legitimacy is $L_{\lambda=0}=0.89$. With the value for the rescaled legitimacy, the simulations are repeated with the general parameters shown in Table 2.

As seen in Figure 6(a), in all distributions, the maximum frequency takes place for wait times of the order of 50. In contrast to what is observed in Figure 3(a) with Epstein's legitimacy, cases of heterogeneous savings and $\lambda=0.00$ show the same characteristic as cases of homogeneous saving, having a maximum of frequency in standby time 50 . Like what is observed in Figure 3(a), all cases differ in the maximum value of the frequency. Likewise, the higher frequency is obtained when the distribution of savings is heterogeneous. The result of the rescaling produces that the maximum frequency for the Epstein case is greater than those obtained for cases with homogeneous savings. For the outburst sizes distribution (see Figure 6(b)), the case with heterogeneous saving has the largest sizes and frequency of outbursting. The cases where the agents are willing to save have both size and the maximum value of the outburst frequency decreasing for values of decreasing outburst size and the saving factor increases. The maximum frequency in the case studied by Epstein is similar to the maximum of the frequency in the outbursts size for the case with saving factor $\lambda=0.05$, but with an outburst frequency very similar to the heterogeneous case.

In Figure 7(a), the outburst quantity is plotted as a function of the saving parameter with the rescaled legitimacy. With the line with red squares, the quantity of outburst is plotted, while the blue circle corresponds to the value 
TABLE 3: The averages for $\left\langle h_{\lambda}\right\rangle$ and $L_{\lambda}$ for each $\lambda$ obtained with the data generated for Case I.

\begin{tabular}{lccccccccccc}
\hline$\lambda$ & $\left\langle h_{\lambda}\right\rangle$ & $L_{\lambda}$ & $\lambda$ & $\left\langle h_{\lambda}\right\rangle$ & $L_{\lambda}$ & $\lambda$ & $\left\langle h_{\lambda}\right\rangle$ & $L_{\lambda}$ & $\lambda$ & $\left\langle h_{\lambda}\right\rangle$ & $L_{\lambda}$ \\
\hline 0.00 & 0,87 & 0,89 & 0.30 & 0.77 & 0.88 & 0.60 & 0.66 & 0.86 & 0.80 & 0.53 & 0.83 \\
0.05 & 0,85 & 0,89 & 0.35 & 0.76 & 0.88 & 0.65 & 0.63 & 0.85 & 0.81 & 0.51 & 0.82 \\
0.10 & 0,83 & 0,89 & 0.40 & 0.74 & 0.87 & 0.70 & 0.60 & 0.85 & 0.83 & 0.50 & 0.82 \\
0.15 & 0,82 & 0,89 & 0.45 & 0.72 & 0.87 & 0.73 & 0.58 & 0.84 & 0.85 & 0.48 & 0.81 \\
0.20 & 0,80 & 0,88 & 0.50 & 0.70 & 0.87 & 0.75 & 0.57 & 0.84 & 0.87 & 0.46 & 0.80 \\
0.25 & 0,79 & 0,88 & 0.55 & 0.68 & 0.86 & 0.77 & 0.56 & 0.84 & 0.90 & 0.41 & 0.78 \\
\hline
\end{tabular}

obtained with the Epstein model and the green diamond corresponds to the value obtained for the case with heterogeneous saving. In Figure 7(b), the waiting times are shown as a function of the saving parameter with the rescaled legitimacy. With the line with red squares, the time between each outburst is plotted as the blue circle corresponds to the value obtained with the Epstein model and the green diamond corresponds to the value obtained for the case with heterogeneous saving. In the case with $L=0.82$, a continuous decrease in the number of outbursts is observed, and the factor of savings of the agents is increased. Unlike the case with $L=0.82$, when $L=0.89$, the outbursts are not observed from $\lambda=\lambda_{c} \approx 0.25$. Note the sensitivity of the appearance of outburst to the value of legitimacy.

It is observed how the time between each outburst increases as savings increase, and consistently with the outbursts quantity (see Figure $7(\mathrm{~b})$ ), there is a sustained increase in waiting times up to $20 \%$ savings. From the value $\lambda=\lambda_{c} \approx 0.25$, the absence of outbursts in the simulation time is observed.

In Figure 8(a), the outburst sizes are plotted against the savings. The red line shows the average value of the size of the outburst in the range $\left[-2 \sigma_{\lambda}, 2 \sigma_{\lambda}\right]$, where $\sigma_{\lambda}$ is the standard deviation of the maximum values of the outburst sizes for each value of $\lambda$. The magenta and cyan lines show the average value of the size of the outbursts above and below the range $\left[-2 \sigma_{\lambda}, 2 \sigma_{\lambda}\right]$, respectively. The blue circle corresponds to the average size of the outbursts obtained for the Epstein model and the diamond corresponds to the average size of the outbursts obtained with heterogeneous lambda. In Figures $8(\mathrm{~b}), 8(\mathrm{c})$, and $8(\mathrm{~d})$, the average value of the size of the outbursts with their corresponding error bars, obtained both in the range and above and below the range, respectively, is plotted in detail.

As can be seen in Figure 8, the size of the outburst similarly decreases as the number of outbursts (see Figure 7); in the absence of outburst it is from $\lambda=0.25$. Again we have the appearance of three regimes of the maxima in each outburst as in Figure 5, the maxima of lower intensity (see Figure $8(\mathrm{~d})$ ), and the outbursts with maximum values above the range $\left[-2 \sigma_{\lambda}, 2 \sigma_{\lambda}\right]$ (see Figure $\left.8(\mathrm{c})\right) \lambda=[0.00,0.05]$.

By introducing the money-dependent hardship parameter, the outburst quantity produced in the system continuously decreases as agent savings increase as can be seen in Figure 4(a). This behavior is characteristic of a second-order phase transition, according to the classification criteria found in the literature [34]. As the outbursts decrease, the regularity with which they occur also decreases, which implies an increase in the waiting times between the outbursts; the results can be observed in Figure 4(b). In the same way that the outburst quantity decreases with the increase in savings in the system, the outburst size also decreases, which can be seen in Figure 5(b). Note that in Figures 5(c) and 5(d) there are both outbursts on average as well as under average, which indicates that there are different dynamics associated with outbursts. There are events where active agents have better conditions in their neighborhood to join a protest generating a burst above average. On the other hand, outbursts below average respond to smaller outbursts that come after a major outburst, so it can be interpreted as a replica of the previous main outburst. These subsequent events are due to the fact that at the end of the main outburst the most active agents are not captured, which are reactivated by improving the conditions in their neighborhood, producing a second successive outburst.

These results can be observed at the macroscopic level by looking at the distributions of the wait times in Figure 3(a) as well as in the outburst sizes distribution Figure 3(b). In the case of waiting times, we can see that as the savings increase in the system, the value associated with the more frequent time is increasing, as well as a deformation of the distribution due to the fact that the outburst decreases in both their regularity and quantity. For the outburst sizes distribution, it is observed that the most frequent size decreases as savings increase, which causes a deformation of the distribution both in its more frequent values and also in its form, showing how the dynamics of the outbursts change as they cease to occur.

The results obtained for simulations with $L=0.89$ were the same results described for $L=0.82$. However, the disappearance of the outbursts with $L_{\lambda}=0.89$ occurs for $\lambda=\lambda_{c} \approx 0.25$ unlike the case with $L_{E}=0.82$ occurring for $\lambda=\lambda_{c} \approx 0.90$. This is because increasing legitimacy, $L$, decreases the grievance of each agent, which generates a decrease in the group of agents that could change their status given the conditions in their neighborhood. As a result, the activity of active agents can be reduced by imposing fewer savings in the system. That is to say that the more legitimate the system is, the less we need to save as a redistributive factor to reduce social protests.

Figure 9 shows the power spectrum of a number of active agents during a simulation with $L=0.82$, using the Fourier transform. In blue we have the result obtained for the model of Epstein and in green the one corresponding to the simulation with heterogeneous saving. In red 


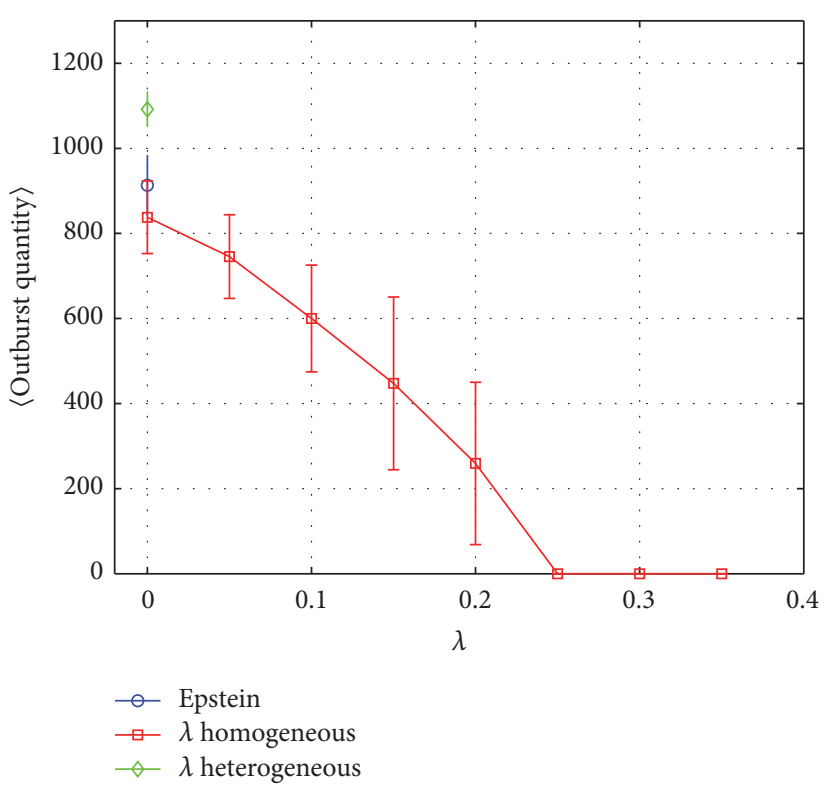

(a)

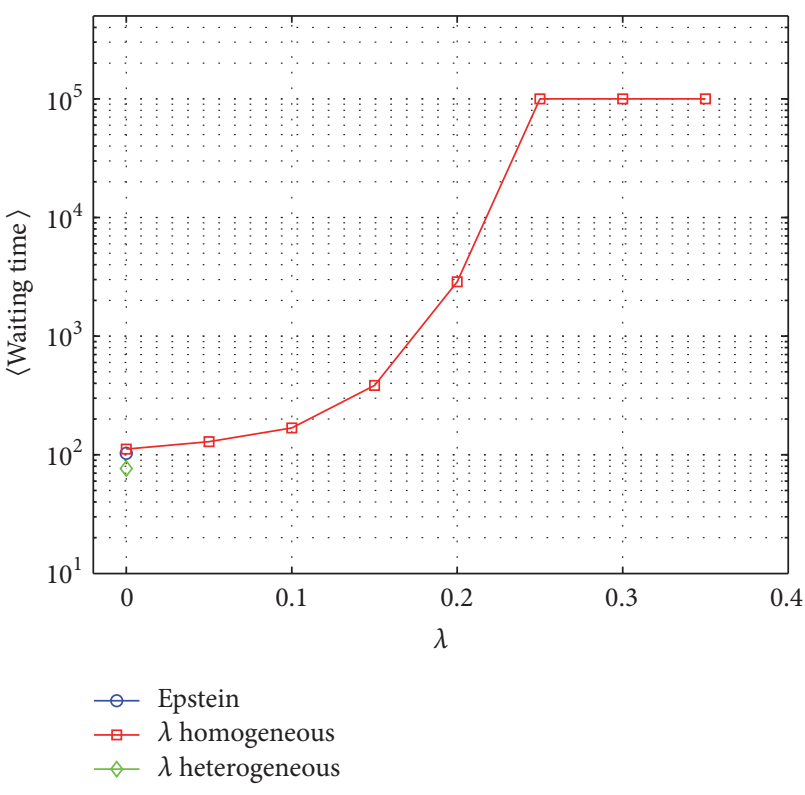

(b)

FIGURE 7: (a) The outburst quantity and (b) the waiting times are shown as a function of the saving parameter with the rescaled legitimacy. The line with red squares shows the time between each outburst for homogeneous $\lambda$. The blue circle corresponds to the value obtained with the Epstein model and the green diamond corresponds to the value obtained for the case with heterogeneous saving.

the cases for the homogeneous saving model are plotted with $\lambda=[0.00,0.70,0.81,0.82,0.87,0.90]$, respectively. The dashed lines show the region of the frequency range used to obtain the slope of the decay of the energy cascade in the inertial range. Spectra are observed to occur in the same frequency range $\left[10^{-4}, 10^{0}\right]$. In the low frequency range, an increase in energy is observed as a consequence of the increase of the active agents in the system. The second range, at intermediate frequencies, presents energy cascades characteristic of turbulent phenomena [35].

In Figure 9(a), the spectral power of the number of active agents for the studied cases is shown. It is observed that the spectrum obtained for the Epstein model develops near $10^{\circ}$ in the spectral power range, whereas for the heterogeneous saving model it is of similar characteristics as the cascade obtained for the saving model with $\lambda=0.00$ since they are developed in the same ranges of spectral power. This indicates that the system in these cases behaves similarly and has the same amount of energy. Comparing these cases with the spectrum of the Epstein model, we verified that the dynamics in these models are different from the dynamics of the punctuated equilibrium, as we observed in Figure 5. This behavior remains for the model with homogeneous saving until $\lambda=0.70$, to arrive at the case with $\lambda=0.81$ where the spectrum develops in the same ranges of the spectral power for the case of Epstein model. This indicates that the spectral power for the cases with savings between 0.70 and 0.81 decreases until reaching the spectral power associated with the dynamics of the punctuated equilibrium. Then, it is observed that for the model with homogeneous savings between 0.82 and 0.87 , it decreases rapidly, arriving at the case with $\lambda=0.90$, where the spectrum obtained is characteristic of the white noise. These results are consistent with those shown in Figures 4 and 5, where the absence of outburst is reported near $\lambda=0.87$.

The power spectrum of the number of active agents obtained during a simulation for the case with $L=0.89$ is shown in Figure 10. The blue color corresponds to the power spectrum obtained for the Epstein model; in green color the spectrum of power corresponds to the simulation with heterogeneous saving. In red color, the power spectrum is drawn for the cases for the homogeneous saving model with $\lambda=[0.00,0.05,0.15,0.25,0.40,0.50]$, respectively. The region between the segmented lines shows the frequency range used to obtain the slope of the decay of the energy cascade in the inertial range. This figure shows the same behavior described in Figure 9, but $\lambda=0.05$ resumes the dynamics of the punctuated equilibrium.

Figure 11(a) shows the slope associated with the cascade of the power spectrum as a function of the saving parameter with $L=0.82$ and in Figure 11(b) the results corresponding to the simulations with $L=0.89$ are shown. With red squares the slope is drawn for the model with homogeneous saving; the blue circle corresponds to the value obtained with the Epstein model, while the green diamond is the result for the slope in the model with heterogeneous saving. In this figure, it is observed that the value of the slope obtained for the energy cascade of the Epstein model, for both cases, corresponds to the behavior of power law $-5 / 3$.

In Figure 11(a), it can be seen that the slope value for the heterogeneous model has the same value as for the case with homogeneous savings with $\lambda=0.00$, which is evidence that the dynamics are similar. There are three clear behaviors (states); the first is where the value of the slope for the case 


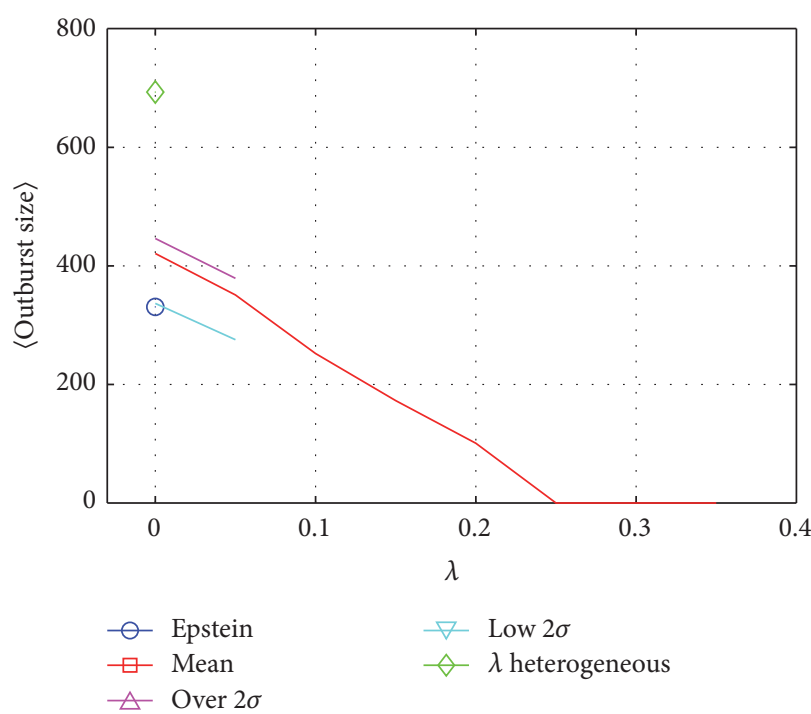

(a)

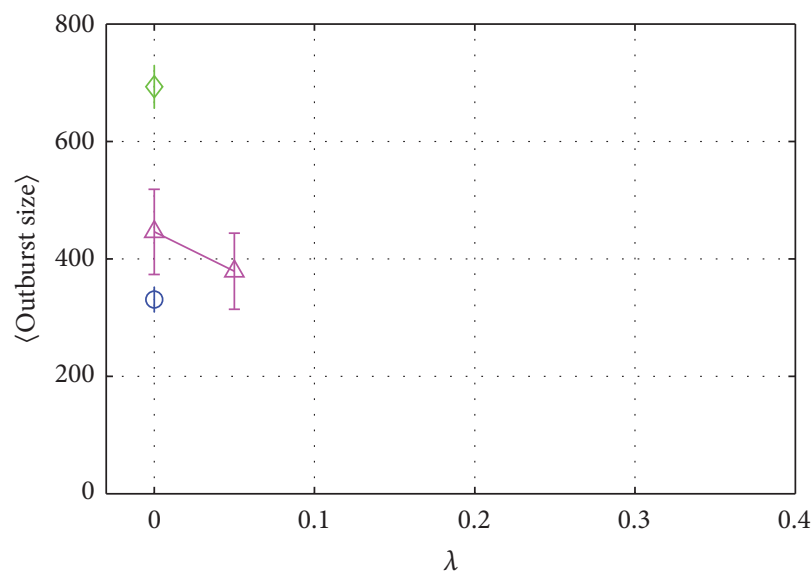

$$
\begin{aligned}
& \curvearrowright \text { Epstein } \\
& \triangle \quad \text { Over } 2 \sigma \\
& \diamond \lambda \text { heterogeneous }
\end{aligned}
$$

(c)

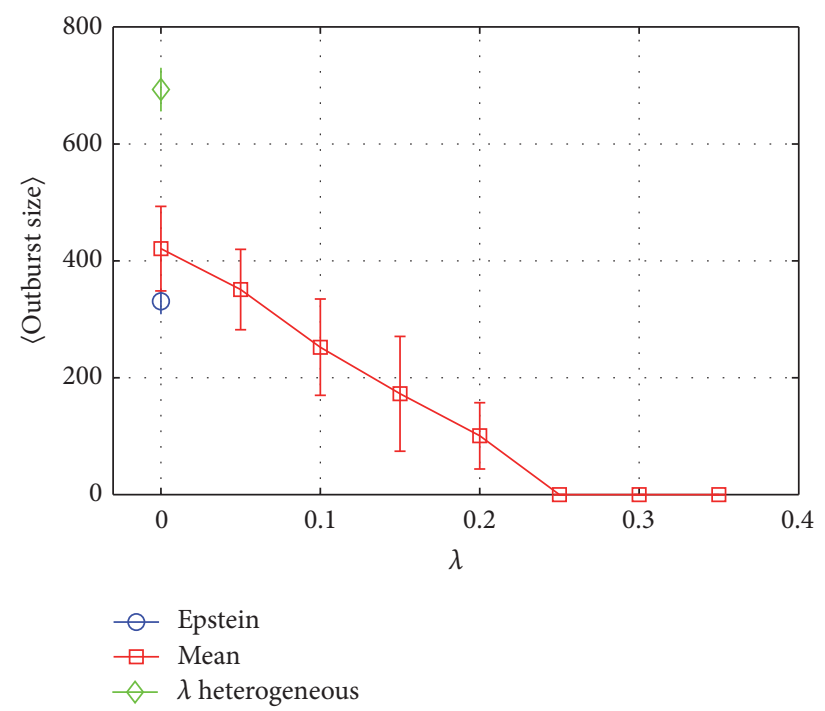

(b)

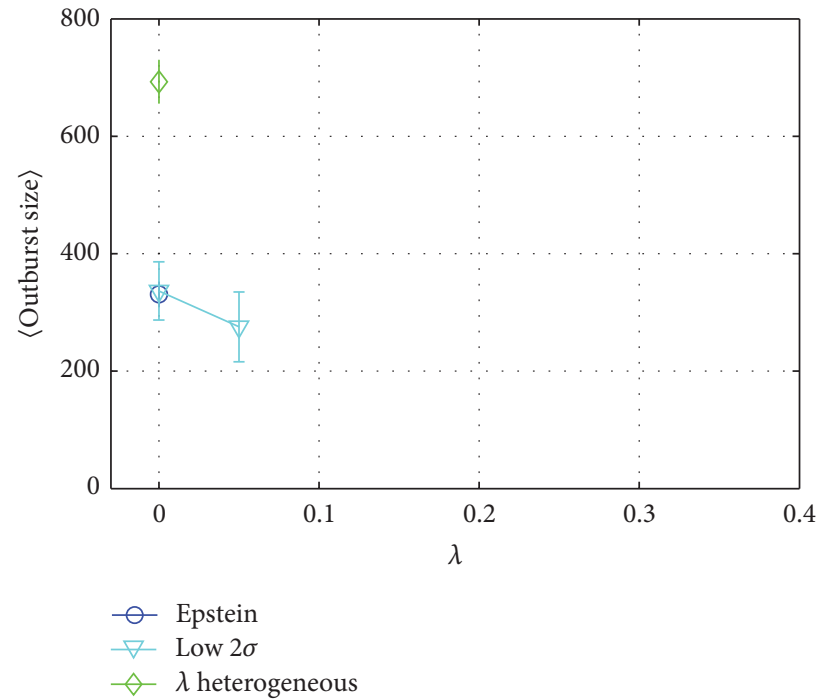

(d)

Figure 8: (a) The outburst sizes depending on the savings. The lines with red squares show the average value of the size of the outburst in the range $\left[-2 \sigma_{\lambda}, 2 \sigma_{\lambda}\right]$, where $\sigma_{\lambda}$ is the standard deviation of the maximum values of the outburst sizes for each value of $\lambda$. The magenta and cyan lines show the average value of the size of the outbursts above and below the range $\left[-2 \sigma_{\lambda}, 2 \sigma_{\lambda}\right]$, respectively. The blue circle corresponds to the average size of the outbursts obtained for the Epstein model and the diamond the average size of the outbursts obtained with heterogeneous lambda. In Figures (b), (c), and (d) the average value of the size of the outbursts with their corresponding error bars, obtained both in the range $\left[-2 \sigma_{\lambda}, 2 \sigma_{\lambda}\right]$ and over and under the range, respectively.

with homogeneous saving stays almost constant up to $\lambda=$ 0.60 , and then there is a decrease in the value of slopes in a sustained way up to $\lambda=0.81$, where its value is close to the value obtained with the Epstein model, which indicates that the energy and the dynamics of the punctuated equilibrium are recovered. Then for values between 0.82 and 0.90 , a progressive increase in the value of the slopes is observed until values close to zero are reached, indicating that the slope disappears and the spectrum associated with the white noise is obtained, as we can see in Figure 10(f). In the first state, the spectrum has a behavior of type $1 / f$ own log-normal distributions, in the second state the spectrum is turbulent, and in the last state there is no dependence of $f$.

In Figure 11(b), it can be seen that the slope value obtained for the heterogeneous model is larger than for the homogeneous savings model of $\lambda=0.00$. Then it can be seen that, for $\lambda=0.05$, the slope value is similar to that obtained for the Epstein model, which indicates the recovery of the punctuated equilibrium. Then, from $\lambda=0.10$, the slope value begins to decrease to zero in the lambda values of 0.45 and 0.50 , where the power spectrum already has white noise characteristics. 


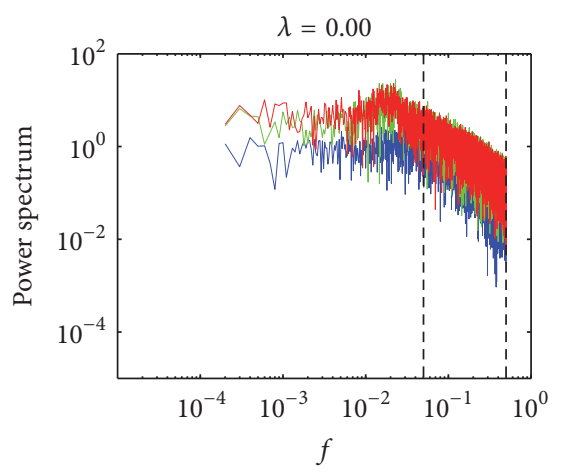

(a)

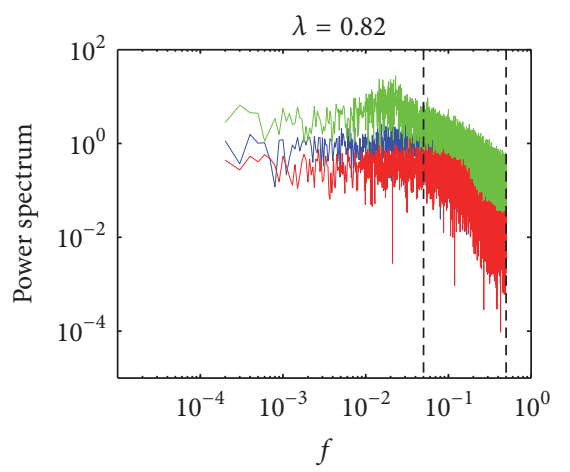

(d)

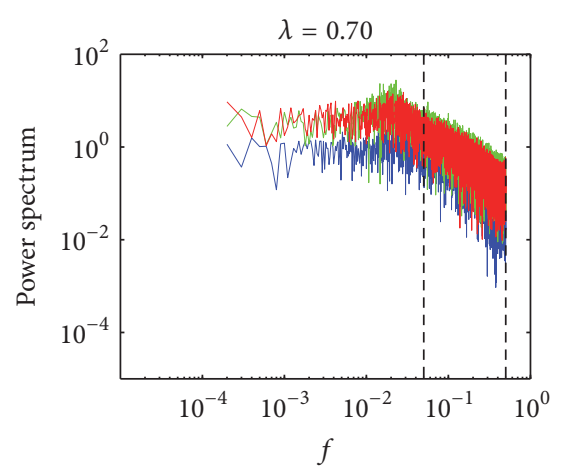

(b)

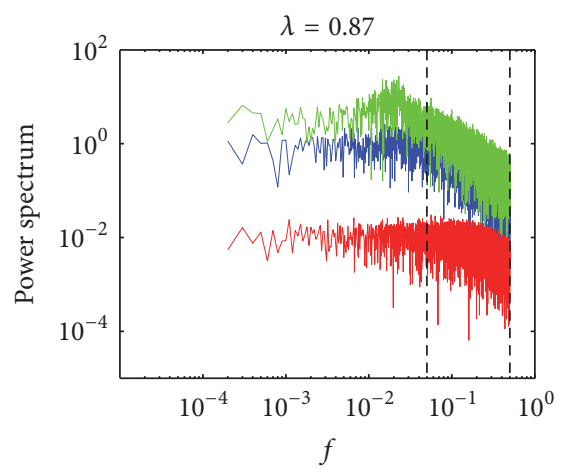

(e)

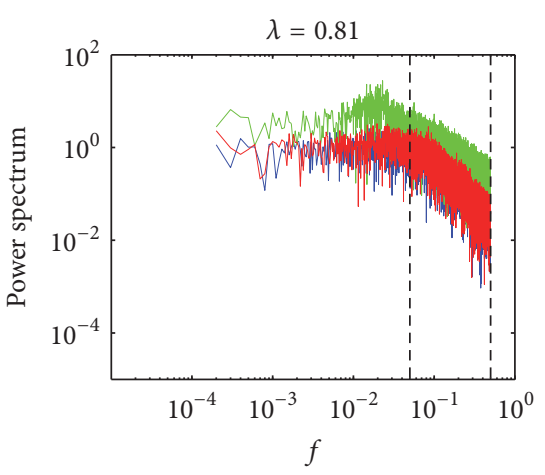

(c)

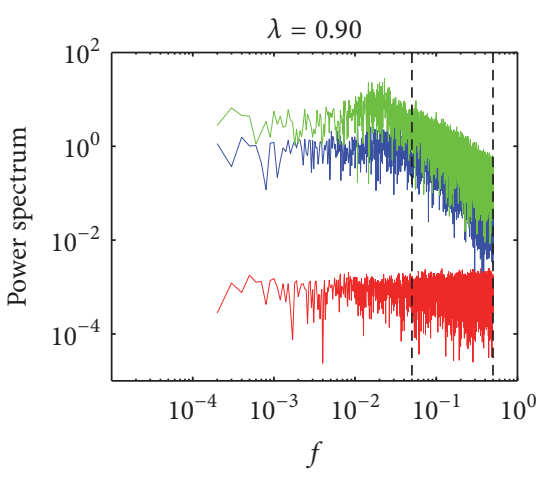

(f)

FIGURE 9: The power spectrum of a number of active agents during a simulation with $L=0.82$, using the Fourier transform. In blue we have the result obtained for the model of Epstein and in green the one corresponding to the simulation with heterogeneous saving. In red the cases for the homogeneous saving model are plotted with $\lambda=[0.00,0.70,0.81,0.82,0.87,0.90]$, respectively. The dashed lines show the region of the frequency range used to obtain the slope of the decay of the energy cascade in the inertial range.

These results show the existence of three characteristic regimes that depend on the savings in the system. The first scheme is given between $\lambda=0.00$ and $\lambda=0.70$ when $L=0.82$ and for $\lambda=0.00$ with $L=0.89$. This is characterized by a wave of successive protests, a product of the replicas generated by a lot of active agents in the system. From the power spectrum, it is observed that there is a greater amount of energy in the system in this scheme. The second regime is punctuated equilibrium, characteristic of the Epstein model. This phase of the system is obtained by increasing the savings to $\lambda=0.81$, when $L=0.82$ and for $\lambda=0.05$ with $L=$ 0.89 . Evidence of this is the $-5 / 3$ power law that appears in the power spectrum. The last regime is the absence of burst, between $\lambda=0.87$ and $\lambda=0.90$ when $L=0.82$ and from $\lambda=0.40$ with $L=0.89$. This is characterized by having activity under the threshold defined for an outburst, which is observed in the power spectrum as white noise. These regimes account for emerging phenomena associated with system savings levels and define scales of development characteristic of social conflicts understood as a complex system [7].

\section{Conclusions}

In this paper, we study the influence of the distribution of money on the emergence of social mobilizations using the dynamics of Epstein's civil violence model conditioned through the hardship parameter distributed according to the distribution of money with savings $[28,29]$ between agents. Our experiments show that hardship is one of the determining factors for the model of civil violence and this model, so its association to the distribution of money is fundamental to establish a variation of the dynamics.

In the cases we have analyzed, we find that the outbursts quantity and the number of active agents are decreasing as the saving factor increases. Let us assume the average quantity of outbursts as an order parameter. This determines a change of a phase characterized by very regular outbursts of protest to another one characterized by the absence of outbursts. The order parameter varies continuously vanishing at the critical point for a critical value of the saving parameter $\left(\lambda_{c}\right)$. This behavior is characteristic of a second-order phase transition, according to the classification criteria found in the literature [34]. Moreover, the critical value of the saving parameter, $\lambda_{c}$, depends on the legitimacy parameter, $L$. Our results show that when legitimacy increases, the outbursts disappear with a much lower critical value of the saving parameter. This also indicates that the more legitimacy the authority has in the system, the fewer savings are needed as a redistributive factor to reduce social protests.

These results show three characteristic regimes that depend on the savings in the system. The first regime is 


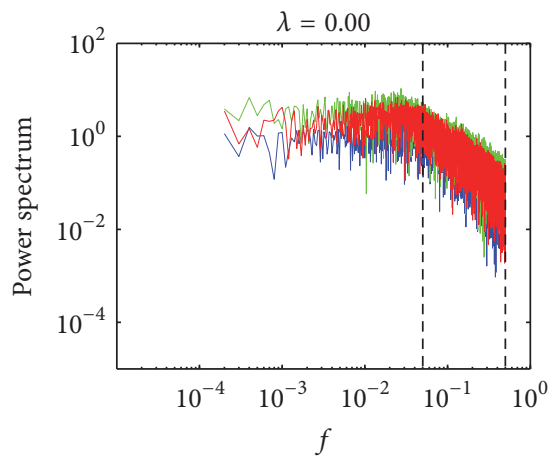

(a)

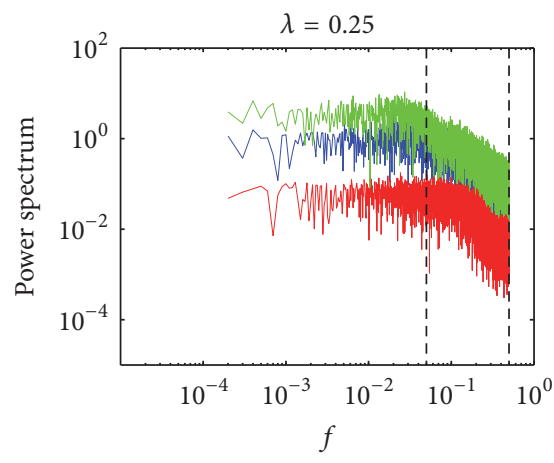

(d)

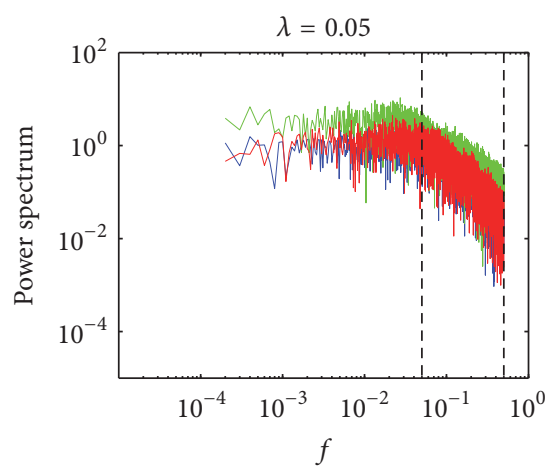

(b)

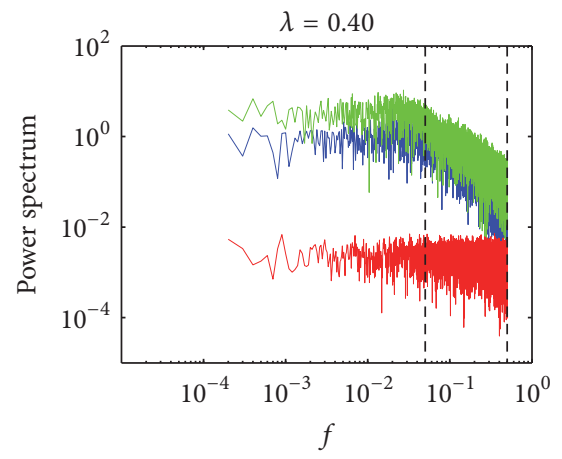

(e)

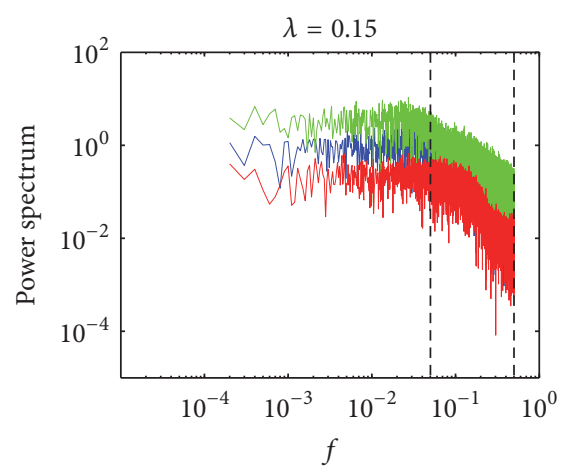

(c)

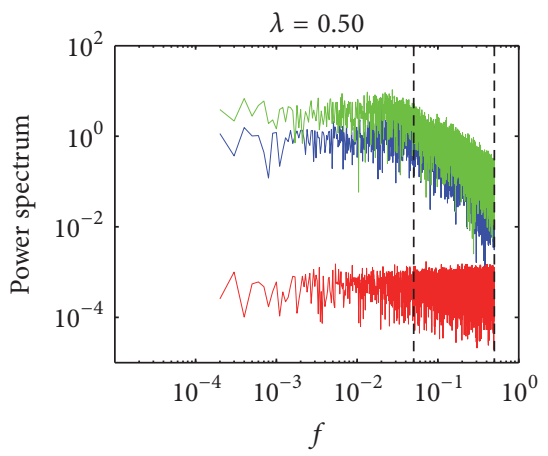

(f)

FIgURE 10: The power spectrum of a number of active agents obtained during a simulation for the case with $L=0.89$ is shown. The blue color corresponds to the power spectrum obtained for the Epstein model; in green color the spectrum of power corresponds to the simulation with heterogeneous saving. In red color, the power spectrum is drawn for the cases for the homogeneous saving model with $\lambda=[0.00,0.05,0.15,0.25,0.40,0.50]$, respectively. The region between the segmented lines shows the frequency range used to obtain the slope of the decay of the energy cascade in the inertial range.

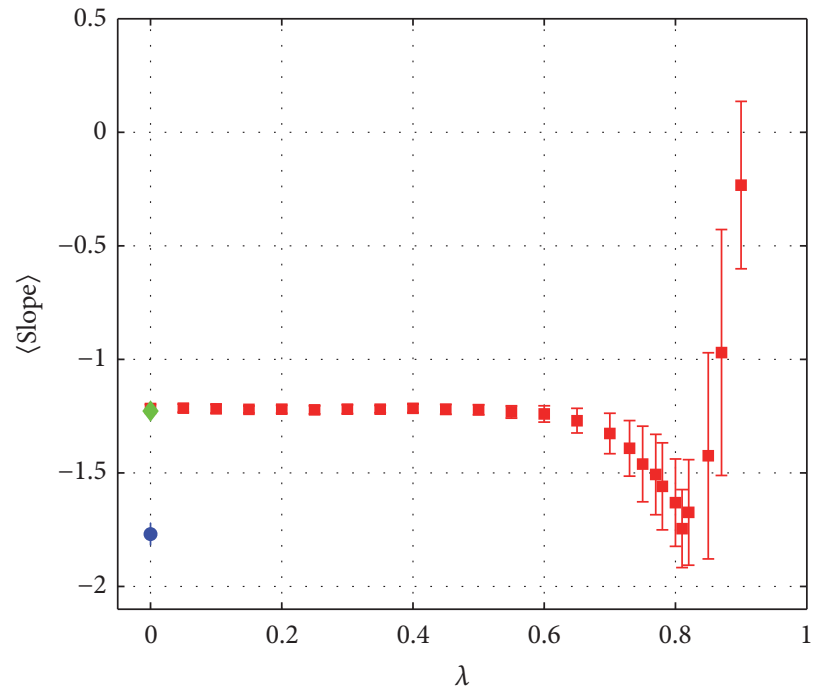

- Epstein

$\lambda$ homogeneous

$\wedge \lambda$ heterogeneous

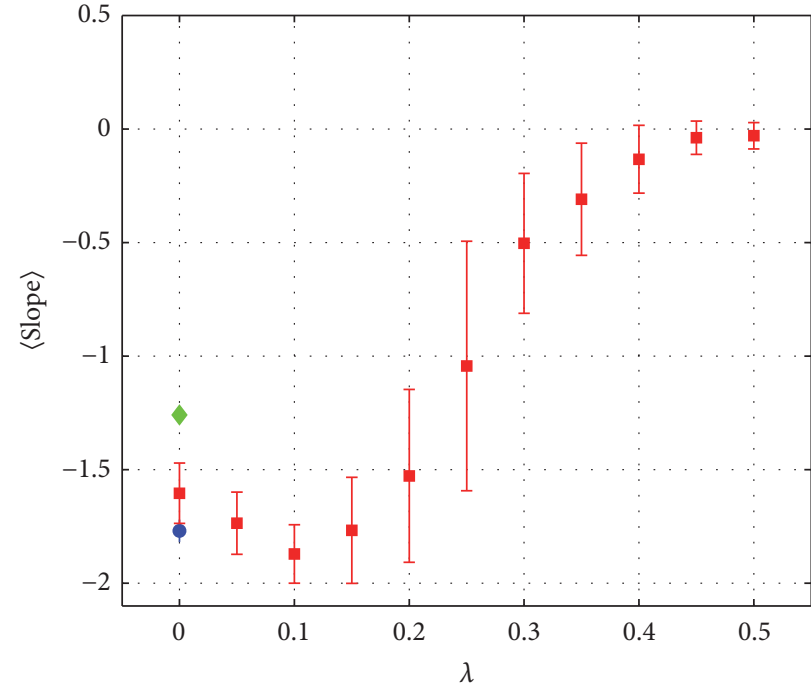

Epstein

$\lambda$ homogeneous

$\lambda$ heterogeneous

(a)

(b)

FIGURE 11: (a) shows the slope associated with the cascade of the power spectrum as a function of the saving parameter with $L=0.82$ and in (b) the results corresponding to the simulations with $L=0.89$ are shown. With red squares, the slope is drawn for the model with homogeneous saving, and the blue circle corresponds to the value obtained with the Epstein model, while the green diamond is the result for the slope in the model with heterogeneous saving. 
characterized by a wave of successive protests and more energy is observed in the system. The second regime is punctuated equilibrium, characteristic of the Epstein model. The last regime is the absence of outbursts. These regimes account for phenomena associated with the savings levels of the system and define scales of development characteristic of social conflicts understood as a complex system [7].

The importance of this model is to provide a tool to understand one of the edges that characterize social protest, which adds to other efforts made [9-11, 13] to describe this phenomenon from other points of view. Undoubtedly, to understand this phenomenon in depth it is possible to establish more parameters that determine unhappiness, such as emotional, cultural, or other factors, which allows for more realistic approaches to the cost of making the model more complex, which will be addressed in future works.

\section{Conflicts of Interest}

The authors declare no conflicts of interest regarding the publication of this manuscript.

\section{References}

[1] P. Ball, "Masa Crtica: Cambio, Caos y Complejidad," in Coleccion Noema, Fondo De Cultura Economica USA, San Diego, Calif, USA, 2010.

[2] J. M. Epstein, "Agent-based computational models and generative social science," Complexity, vol. 4, no. 5, pp. 41-60, 1999.

[3] T. C. Schelling, "Models of segregation," The American Economic Review, vol. 59, no. 2, pp. 488-493, 1969.

[4] R. Axelrod, "The dissemination of culture: a model with local convergence and global polarization," Journal of Conflict Resolution, vol. 41, no. 2, pp. 203-226, 1997.

[5] J. M. Epstein and R. Axtell, Growing Artificial Societies: Social Science from the Bottom Up. A Bradford book, Brookings Institution Press, 1996.

[6] J. M. Epstein, "Generative Social Science: Studies in AgentBased Computational Modeling," in Princeton Studies in Complexity, Princeton University Press, 2006.

[7] L. Carlos, H. Coelho, and R. J. Lopes, "Agent-based modeling of social conflict, civil violence and revolution: state-of-the-artreview and further prospects," in Proceedings of the Eleventh European Workshop on Multi-Agent Systems (EUMAS), pp. 124138, Toulouse, France, 2013.

[8] J. M. Epstein, "Modeling civil violence: An agent-based computational approach," Proceedings of the National Acadamy of Sciences of the United States of America, vol. 99, no. 3, pp. 72437250, 2002.

[9] J. Kim and R. Hanneman, "A Computational Model of Worker Protest," Journal of Artificial Societies and Social Simulation, vol. 14, no. 3, 2011.

[10] M. Fonoberova, V. A. Fonoberov, I. Mezic, J. Mezic, and P. Jeffrey Brantingham, "Nonlinear dynamics of crime and violence in Urban settings," Journal of Artificial Societies and Social Simulation, vol. 15, no. 1, 2012.

[11] R. Bhavnani and H. J. Choi, "Modeling civil violence in Afghanistan: Ethnic geography, control, and collaboration," Complexity, vol. 17, no. 6, pp. 42-51, 2012.
[12] C. K. Goh, H. Y. Quek, K. C. Tan, and H. A. Abbass, "Modeling civil violence: An evolutionary multi-agent, game theoretic approach," in Proceedings of the 2006 IEEE Congress on Evolutionary Computation, CEC 2006, pp. 1624-1631, July 2006.

[13] L. Carlos, R. J. Lopes, and C. Helder, "On legitimacy feedback mechanisms in agent-based modeling of civil violence," International Journal of Intelligent Systems, vol. 31, no. 2, pp. 106-127, 2016.

[14] D. Stauffer, "Introduction to statistical physics outside physics," Physica A: Statistical Mechanics and its Applications, vol. 336, no. 1-2, pp. 1-5, 2004.

[15] A. Hansen, "Grand challenges in interdisciplinary physics," Frontiers of Physics, vol. 2, article A058, 2014.

[16] Y. Holovatch, R. Kenna, and S. Thurner, "Complex systems: physics beyond physics," European Journal of Physics, vol. 38, no. 2, 2017.

[17] R. N. Mantegna and H. E. Stanley, An Introduction to Econophysics: Correlations and Complexity in Finance, Cambridge University Press, Cambridge, UK, 2000.

[18] V. M. Yakovenko and J. B. Rosser, "Colloquium: Statistical mechanics of money, wealth, and income," Reviews of Modern Physics, vol. 81, no. 4, pp. 1703-1725, 2009.

[19] A. Chakraborti, I. Muni Toke, M. Patriarca, and F. Abergel, "Econophysics review: I. Empirical facts," Quantitative Finance, vol. 11, no. 7, pp. 991-1012, 2011.

[20] A. Chakraborti, I. Muni Toke, M. Patriarca, and F. Abergel, "Econophysics review: II. Agent-based models," Quantitative Finance, vol. 11, no. 7, pp. 1013-1041, 2011.

[21] B. K. Chakrabarti, A. Chakraborti, and A. Chatterjee, Econophysics and Sociophysics: Trends and Perspectives, Wiley, 2007.

[22] S. Galam, "Sociophysics: a physicist's modeling of psychopolitical phenomena," in Understanding Complex Systems, Springer, New York, NY, USA, 2012.

[23] C. Castellano, S. Fortunato, and V. Loreto, "Statistical physics of social dynamics," Reviews of Modern Physics, vol. 81, no. 2, pp. 591-646, 2009.

[24] S. Galam, Y. Gefen, and Y. Shapir, "Sociophysics: A new approach of sociological collective behaviour. I. meanbehaviour description of a strike," The Journal of Mathematical Sociology, vol. 9, no. 1, pp. 1-13, 1982.

[25] S. Galam, "Application of statistical physics to politics," Physica A: Statistical Mechanics and its Applications, vol. 274, no. 1, pp. 132-139, 1999.

[26] S. Galam, "Sociophysics: A personal testimony," Physica A: Statistical Mechanics and its Applications, vol. 336, no. 1-2, pp. 49-55, 2004

[27] D. Stauffer, "Social applications of two-dimensional Ising models," American Journal of Physics, vol. 76, no. 4-5, pp. 470-472, 2008.

[28] A. Chakraborti and B. Chakrabarti, "Statistical mechanics of money: how saving propensity affects its distribution," The European Physical Journal B, vol. 17, no. 1, pp. 167-170, 2000.

[29] A. Chatterjee, B. K. Chakrabarti, and S. S. Manna, "Pareto law in a kinetic model of market with random saving propensity," Physica A: Statistical Mechanics and its Applications, vol. 335, no. 1-2, pp. 155-163, 2004.

[30] P. Bak and K. Sneppen, "Punctuated equilibrium and criticality in a simple model of evolution," Physical Review Letters, vol. 71, no. 24, pp. 4083-4086, 1993.

[31] M. Patriarca, A. Chakraborti, and K. Kaski, "Statistical model with a standard $\Gamma$ distribution," Physical Review E: Statistical, 
Nonlinear, and Soft Matter Physics, vol. 70, no. 1, article 016104, 2004.

[32] A. Dragulescu and V. M. Yakovenko, "Statistical mechanics of money," The European Physical Journal B-Condensed Matter and Complex Systems, vol. 17, no. 4, pp. 723-729, 2000.

[33] A. Dragulescu and V. M. Yakovenko, "Exponential and powerlaw probability distributions of wealth and income in the United Kingdom and the United States," Physica A: Statistical Mechanics and its Applications, vol. 299, no. 1-2, pp. 213-221, 2001.

[34] S. Parongama and B. K. Chakrabarti, Sociophysics: An Introduction, Oxford University Press, 2014.

[35] U. Frisch and A. N. Kolmogorov, Turbulence: The Legacy of A. N. Kolmogorov, Cambridge University Press, 1995. 


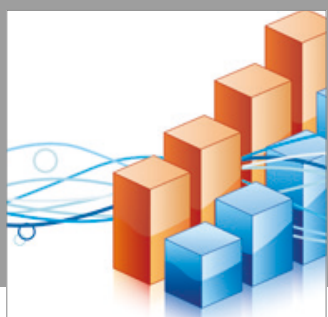

Advances in

Operations Research

vatersals

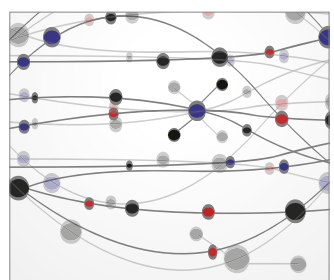

\section{The Scientific} World Journal
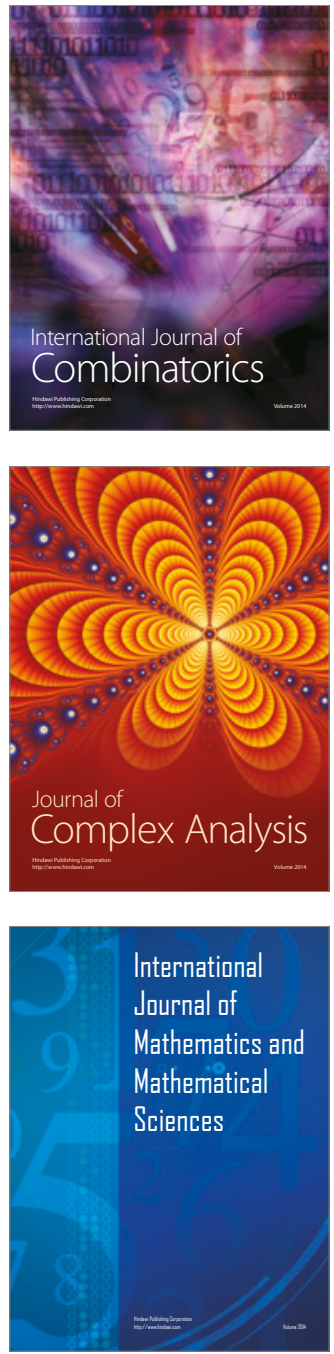
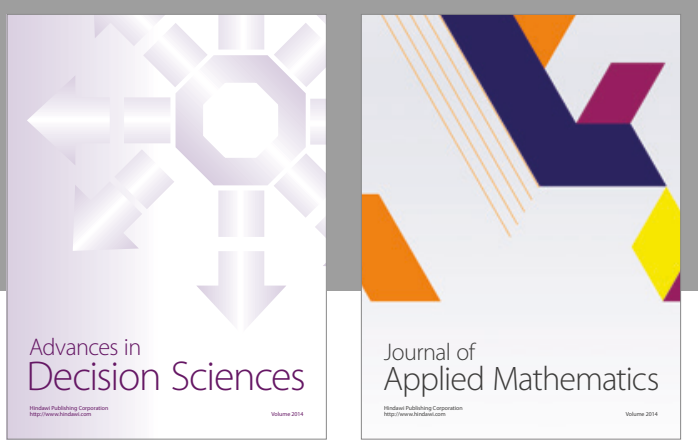

Algebra

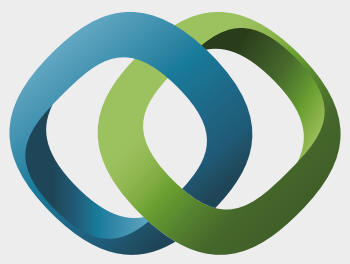

\section{Hindawi}

Submit your manuscripts at

https://www.hindawi.com
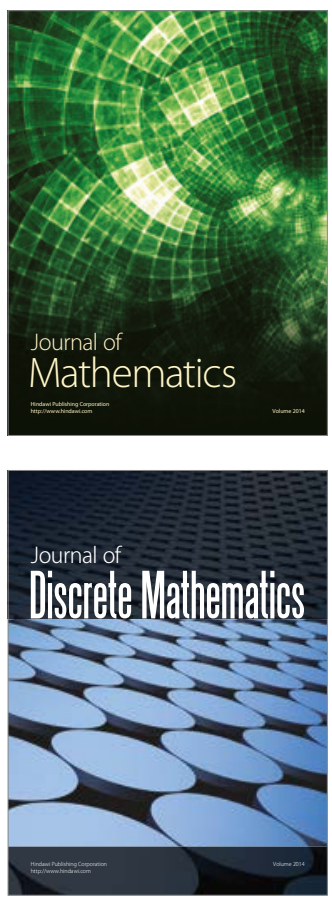

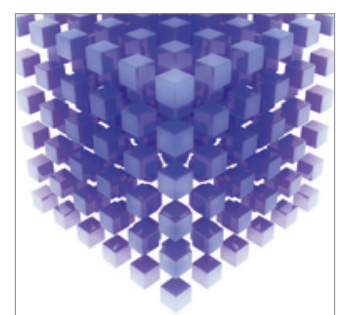

Mathematical Problems in Engineering
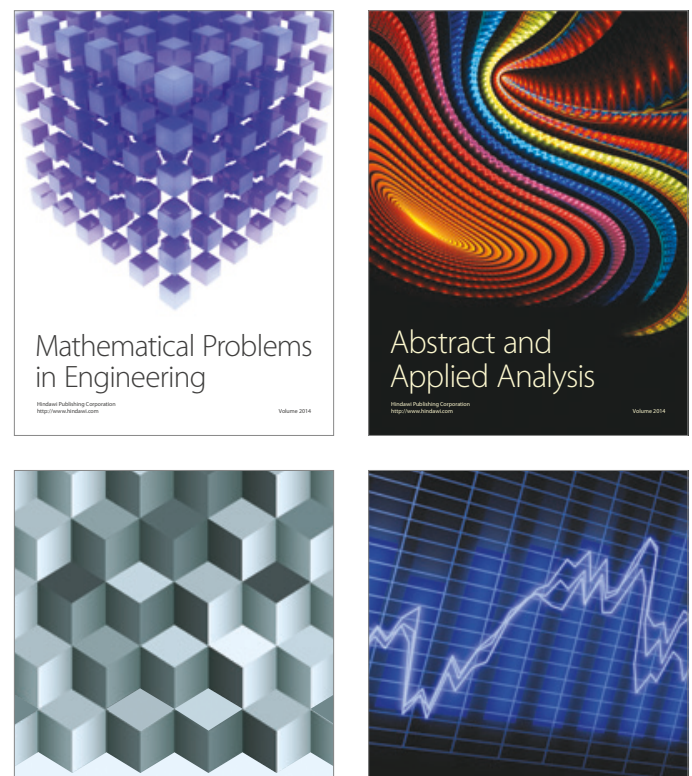

Journal of

Function Spaces

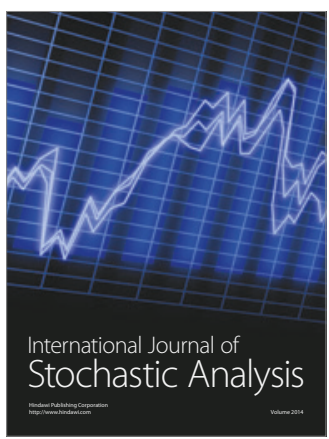

Probability and Statistics
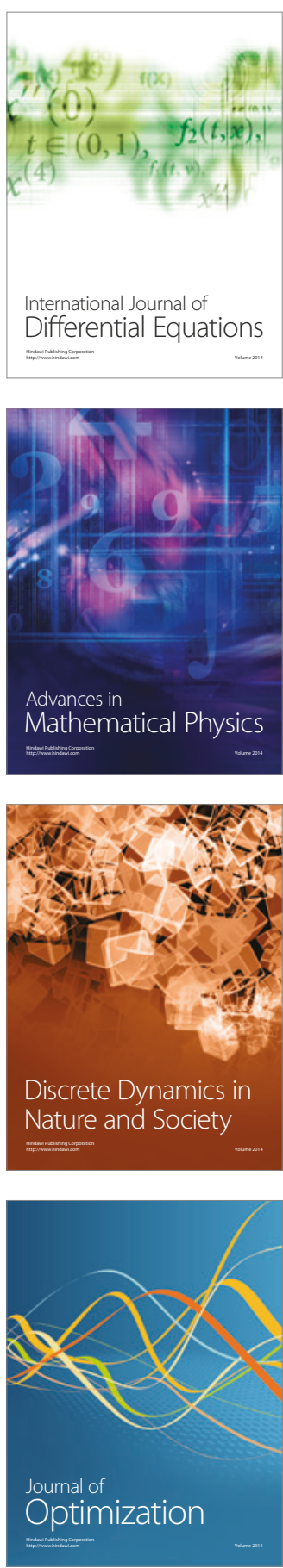